\title{
Filterbank Optimization with Convex Objectives and the Optimality of Principal Component Forms
}

\author{
Sony Akkarakaran, Student Member, IEEE, and P. P. Vaidyanathan, Fellow, IEEE
}

\begin{abstract}
This paper proposes a general framework for the optimization of orthonormal filterbanks (FBs) for given input statistics. This includes as special cases, many recent results on FB optimization for compression. It also solves problems that have not been considered thus far. FB optimization for coding gain maximization (for compression applications) has been well studied before. The optimum FB has been known to satisfy the principal component property, i.e., it minimizes the mean-square error caused by reconstruction after dropping the $P$ weakest (lowest variance) subbands for any $P$. In this paper, we point out a much stronger connection between this property and the optimality of the FB. The main result is that a principal component FB (PCFB) is optimum whenever the minimization objective is a concave function of the subband variances produced by the FB. This result has its grounding in majorization and convex function theory and, in particular, explains the optimality of PCFBs for compression. We use the result to show various other optimality properties of PCFBs, especially for noise-suppression applications. Suppose the FB input is a signal corrupted by additive white noise, the desired output is the pure signal, and the subbands of the FB are processed to minimize the output noise. If each subband processor is a zerothorder Wiener filter for its input, we can show that the expected mean square value of the output noise is a concave function of the subband signal variances. Hence, a PCFB is optimum in the sense of minimizing this mean square error. The above-mentioned concavity of the error and, hence, PCFB optimality, continues to hold even with certain other subband processors such as subband hard thresholds and constant multipliers, although these are not of serious practical interest. We prove that certain extensions of this PCFB optimality result to cases where the input noise is colored, and the FB optimization is over a larger class that includes biorthogonal FBs. We also show that PCFBs do not exist for the classes of DFT and cosine-modulated FBs.
\end{abstract}

\section{INTRODUCTION}

$\mathbf{T}$ HE PROBLEM of optimization of filterbanks (FBs) has been addressed by several authors, and many interesting results have been reported in the last five years. Yet there are a number of optimization problems that have not hitherto been addressed. This paper proposes a general framework for the optimization of orthonormal FBs for given input statistics, which includes many of the known results as special cases. It also produces solutions to a number of problems that have been regarded as difficult or not considered thus far.

A generic signal processing scheme using an $M$-channel uniform perfect reconstruction FB is shown in Fig. 1. The FB is

Manuscript received July 16, 1999; revised September 6, 2000. This work was supported in part by the National Science Foundation under Grant MIP 0703755. The associate editor coordinating the review of this paper and approving it for publication was Dr. Brian Sadler.

The authors are with the Department of Electrical Engineering, California Institute of Technology, Pasadena, CA 91125 USA (e-mail: ppvnath@sys.caltech.edu)

Publisher Item Identifier S 1053-587X(01)00065-4. said to be orthonormal if the $M \times M$ analysis polyphase matrix $\mathbf{E}\left(e^{j \omega}\right)$ is unitary for all $\omega$. The input vector $\mathbf{x}(n)$ is the $M$-fold blocked version of the scalar input $x(n)$. We assume that $\mathrm{x}(n)$ is a zero mean wide sense stationary (WSS) random process with a given power spectral density (psd) matrix $\mathbf{S}_{\mathbf{x x}}\left(e^{j \omega}\right)$. We are also given a class $\mathcal{C}$ of orthonormal uniform $M$-channel $F B$ s. Examples are the class of FBs in which all filters are FIR with a given bound on their order or the class of unconstrained FBs (where there are no constraints on the filters besides those imposed by orthonormality). The problem with which this paper is concerned is that of finding the best $F B$ from $\mathcal{C}$ for the given input statistics $\mathbf{S}_{\mathbf{x x}}\left(e^{j \omega}\right)$ for use in the system of Fig. 1. By "best FB," we mean one that minimizes a well-defined objective function over the class $\mathcal{C}$. To formulate this objective, we need to describe the purpose or application of the FB in Fig. 1 and the nature of the subband processors $P_{i}$. This is done in detail in Section II in a general setting.

\section{A. Relevant Earlier Work}

Consider, in particular, the case where the $P_{i}$ are quantizers for signal compression. We use the model of [14] that replaces the quantizer $P_{i}$ by additive noise of variance $f_{i}\left(b_{i}\right) \sigma_{i}^{2}$. Here

$b_{i} \quad$ number of bits allotted to the quantizer;

$\sigma_{i}^{2} \quad$ its input variance;

$f_{i}$ normalized quantizer function, which is assumed not to depend on the input statistics.

If all quantization noise processes are jointly stationary, we can show that the overall mean square reconstruction error (which is the minimization objective here) is $g=\sum_{i=0}^{M-1}(1 / M) f_{i}\left(b_{i}\right) \sigma_{i}^{2}$. Kirac and Vaidyanathan show [14] that for any given bit allocation $b_{i}$ (not necessarily optimum), the best FB for this problem is a principal component $F B(P C F B)$ for the given class $\mathcal{C}$ and input psd $\mathbf{S}_{\mathbf{x x}}\left(e^{j \omega}\right)$.

The concept of a PCFB is reviewed in Section III-B. PCFBs for certain classes of FBs have been studied earlier. For example, let $\mathcal{C}^{t}$ denote the class of all $M$-channel orthogonal transform coders, i.e., FBs as in Fig. 1 where $\mathbf{E}(z)$ is a constant unitary matrix $\mathbf{T}$. The KLT for the input $\mathbf{x}(n)$ is the transform $\mathbf{T}$ that diagonalizes the autocorrelation matrix of $\mathbf{x}(n)$. It has been well known [12] that the KLT is a PCFB for $\mathcal{C}^{t}$. For the class $\mathcal{C}^{u}$ of all (unconstrained) orthonormal $M$-channel FBs, construction of the PCFB has been studied by Tsatsanis and Giannakis [24] and independently by Vaidyanathan [26]. The goal of [26] was coding gain maximization for compression under the high bitrate quantizer noise model with optimum bit allocation. This model is, in fact, a special case of the one described earlier, where $f_{i}\left(b_{i}\right)=c 2^{-2 b_{i}}$. In another work on PCFBs [25], Unser 
(a)
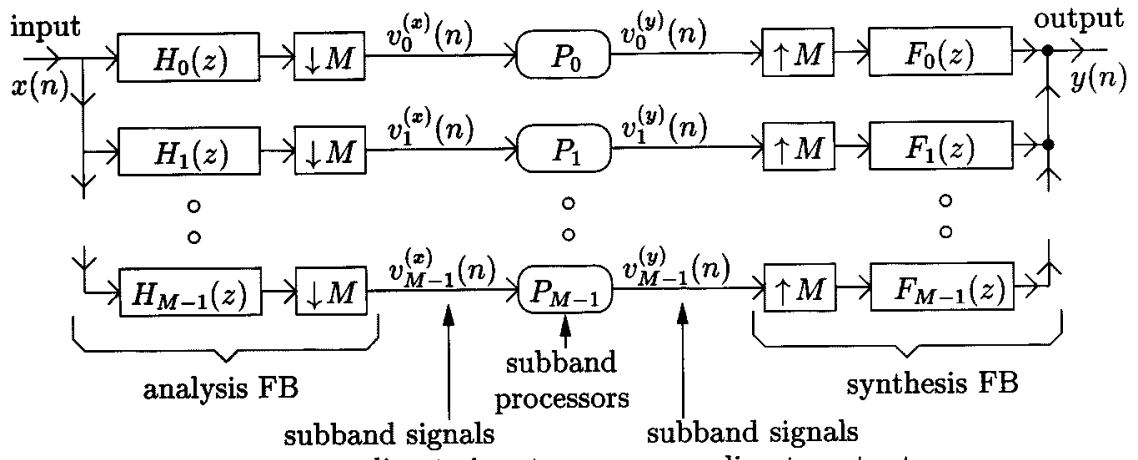

corresponding to input corresponding to output

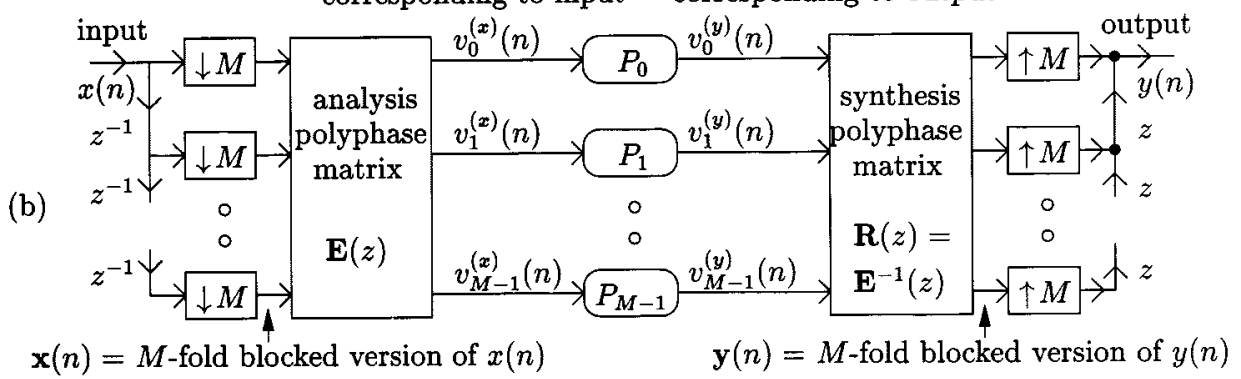

Fig. 1. Generic FB-based signal processing scheme. (a) Analysis and synthesis filters. (b) Polyphase representation.

correctly conjectures their optimality for another family of objective functions of the form $g=\sum_{i=0}^{M-1} h\left(\sigma_{i}^{2}\right)$, where $h$ is any concave function. [This does not include the earlier objective since the $f_{i}\left(b_{i}\right)$ depended on the subband index $i$.] For this family, optimality has been proved by Mallat [17, Th. 9.8, p. 398] using a theorem of Hardy et al. In the present paper, we consider the more general form $g=\sum_{i=0}^{M-1} h_{i}\left(\sigma_{i}^{2}\right)$, where $h_{i}$ are possibly different concave functions. We show optimality of PCFBs for all these objectives. This covers a wider class of applications, as shown in Section VI. It includes the conjecture of [25] (proved in [17]) as a special case where $h_{i} \equiv h$ for all $i$. It also includes the minimization objective of [14] as a special case when $h_{i}(x)=f_{i}\left(b_{i}\right) x$ for all $i$.

FB design for quantization error minimization has also been studied by Moulin et al. [19], [20]. The earlier stated form $g=$ $\sum_{i=0}^{M-1}(1 / M) f_{i}\left(b_{i}\right) \sigma_{i}^{2}$ of the error requires modification for biorthogonal FBs. In an important paper [20], Moulin et al. study the minimization of this modified objective over the class of all (unconstrained) biorthogonal FBs for a broad class of $f_{i}\left(b_{i}\right)$. The authors examine the role of the properties of the PCFB for the unconstrained orthonormal FB class $\mathcal{C}^{u}$ in this problem. It is also claimed that pre and post filters around such a PCFB yield the optimal solution. In [19], an algorithm is proposed for PCFB design for a certain class of FIR orthonormal FBs. It involves a compaction filter design followed by a KLT matrix completion and will produce the PCFB (which is known to maximize coding gain) if it exists. However, it is shown numerically that the designed filters do not always optimize the coding gain (thus showing that in fact the PCFB does not exist). The present paper studies the geometric structure of the optimization search space and thereby reveals several new optimality properties of PCFBs, especially those connected with noise reduction. Preliminary results of this work have been presented in [1] and [2].

\section{B. Main Aims of This Paper}

This paper points out a strong connection between orthonormal FB optimization and the principal component property. The main message is as follows. Let $\sigma_{i}^{2}$ denote the variance of the $i$ th subband signal. To every FB in the given class $\mathcal{C}$, there then corresponds a set of subband variances $\sigma_{i}^{2}$. The PCFB for $\mathcal{C}$, if it exists, is the optimum $F B$ in $\mathcal{C}$ for all problems in which the minimization objective can be expressed as a concave function of the subband variance vector $\mathbf{v} \triangleq\left(\sigma_{0}^{2}, \sigma_{1}^{2}, \cdots, \sigma_{M-1}^{2}\right)^{T}$.

This result has its grounding in majorization and convexity theory and will be elaborated in detail in later sections. It shows PCFB optimality for all objectives of the form $g=\sum_{i=0}^{M-1} h_{i}\left(\sigma_{i}^{2}\right)$, where $h_{i}$ are any concave functions. For orthonormal FBs, this general form includes, as special cases, all the objectives mentioned earlier. We show how such concave objectives arise in many other situations besides coding gain maximization, especially those connected with noise suppression. Suppose the FB input is a signal buried in noise, and the system of Fig. 1 aims to improve the signal-to-noise ratio (SNR). We consider the case where each subband processor $P_{i}$ is a zeroth-order Wiener filter. We show that under suitable assumptions on the signal and noise statistics, the problem of FB optimization for such a scheme reduces to the minimization of a concave function of the subband variance vector. Therefore, PCFBs, if they exist, are optimal for such a scheme. PCFB optimality continues to hold even with certain other types of subband processors for noise reduction, although these are of no serious practical interest. Thus, we have a general problem formulation (Section II) and a unified theory of optimal FBs (Section III), which simultaneously explains the optimality of PCFBs for progressive transmission (Section III-B), compression (Section IV-C), and noise suppression 
(Section VI). To emphasize the fact that PCFBs do not always exist, we also show in Section V that the classes of DFT and cosine-modulated FBs do not have PCFBs.

\section{Notations}

Superscripts $(*)$ and $(T)$ denote the complex conjugate and matrix (or vector) transpose, respectively, whereas superscript dagger $(\dagger)$ denotes the conjugate transpose. Boldface letters are used for matrices and vectors. Lowercase letters are used for discrete sequences, whereas uppercase letters are used for Fourier transforms. $\mathcal{R}^{M}$ denotes the set of $M$-tuples of real numbers, and $\mathcal{R}_{+}^{M}$ denotes that of $M$-tuples of non-negative real numbers. We denote by $\operatorname{diag}(\mathbf{A})$ the column vector consisting of the diagonal entries of the square matrix $\mathbf{A}$.

\section{PROBlem Formulation}

We are given a class $\mathcal{C}$ of orthonormal uniform $M$-channel $F B s$. Recall that an FB is fully specified by its analysis polyphase matrix $\mathbf{E}(z)$ or, alternatively, by the ordered set of analysis and synthesis filter pairs $\left(H_{k}(z), F_{k}(z)\right)$, $k=0,1, \cdots, M-1$ (see Fig. 1). We are also given an ordered set of $M$ subband processors $P_{i}, i=0,1, \cdots, M-1$, where $P_{i}$ denotes the processor acting on the $i$ th subband. Specific instances of such $P_{i}$ will be discussed in later sections; in general, each $P_{i}$ is simply a function that maps input sequences to output sequences. The specification of this function may or may not depend on the input statistics.

The system of Fig. 1 is built using an FB in $\mathcal{C}$ and the processors $P_{i}$. In all problems that we consider, this system is aimed at producing a certain desired signal $d(n)$ at the FB output. For example, in context of compression, the processors $P_{i}$ are quantizers, and the desired output equals the input, i.e., $d(n)=x(n)$. In the context of noise reduction, the input $x(n)=s(n)+\mu(n)$, where $\mu(n)$ is additive noise, the desired output $d(n)=s(n)$ (the pure signal), and the $P_{i}$ could, for instance, be Wiener filters. The FB optimization problem involves finding among all FBs in $\mathcal{C}$ the one minimizing some measure of the error signal

$$
e(n) \triangleq d(n)-y(n)
$$

where $y(n)$ is the true FB output. To formulate the error measure, we impose random process models on the FB input $x(n)$ and desired signal $d(n)$. We assume that $\mathbf{x}(n)$, which is the $M$-fold blocked version of $x(n)$ (see Fig. 1), is a WSS vector process with given psd matrix $\mathbf{S}_{\mathbf{x x}}\left(e^{j \omega}\right)$. Equivalently, $x(n)$ is CWSS $(M)$, i.e., wide sense cyclostationary with $M$ as period. ${ }^{1}$

All processes are assumed to be zero mean unless otherwise stated. In all our problems, the $d(n)$ and the $P_{i}$ are such that the error $e(n)$ is also a zero mean $\operatorname{CWSS}(M)$ random process. Thus, we choose as error measure the variance of $e(n)$ averaged over its period of cyclostationarity $M$.

As shown in Fig. 1, we denote by $v_{i}^{(x)}(n)$ the $i$ th subband signal generated by feeding the scalar signal $x(n)$ as input to the FB. If the error $e(n)$ is $\operatorname{CWSS}(M)$, the signals $v_{i}^{(e)}(n), i=$

\footnotetext{
${ }^{1}$ In particular, $x(n)$ could be a WSS process with given power spectrum $S\left(e^{j \omega}\right)$. In this case, $\mathbf{S}_{\mathbf{x x}}\left(e^{j \omega}\right)$ is fully determined from $S\left(e^{j \omega}\right)$ and has the special property of being pseudocirculant.
}

$0,1, \cdots, M-1$ are jointly WSS, and orthonormality of the FB can be used to show that the above-mentioned error measure equals

$$
\frac{1}{M} \sum_{i=0}^{M-1} E\left[\left|v_{i}^{(e)}(n)\right|^{2}\right]
$$

where

$$
v_{i}^{(e)}(n)=v_{i}^{(d)}(n)-v_{i}^{(y)}(n) .
$$

Thus, the processor $P_{i}$ must try to produce an output "as close to" $v_{i}^{(d)}(n)$ as possible, in the sense of minimizing $E\left[\left|v_{i}^{(e)}(n)\right|^{2}\right]$. In many situations to be discussed in detail later, the processors $P_{i}$ are such that

$$
E\left[\left|v_{i}^{(e)}(n)\right|^{2}\right]=h_{i}\left(\sigma_{i}^{2}\right)
$$

Here, $\sigma_{i}^{2}=E\left[\left|v_{i}^{(x)}(n)\right|^{2}\right]$ denotes the variance of $v_{i}^{(x)}(n)$, and $h_{i}$ is some function whose specification depends only on the nature of the processor $P_{i}$ and not on the choice of FB from $\mathcal{C}$. Thus, for such problems, with $\mathbf{v}=\left(\sigma_{0}^{2}, \sigma_{1}^{2}, \cdots, \sigma_{M-1}^{2}\right)^{T}$ denoting the subband variance vector, the objective defined on the class $\mathcal{C}$ becomes

$$
g(\mathbf{v})=\frac{1}{M} \sum_{i=0}^{M-1} h_{i}\left(\sigma_{i}^{2}\right) .
$$

Hence, the minimization objective is purely a function of the subband variance vector. This function $g$ of (4) is fully specified, given the description of the processors $P_{i}$. Let $\mathcal{S}$ denote the set of all subband variance vectors corresponding to all FBs in $\mathcal{C}$. The optimization problem thus reduces to that of finding the minima of the real-valued function $g$ on the set $\mathcal{S}$. We will hence refer to $\mathcal{S}$ as the optimization search space.

In later sections, we show that for a number of FB-based signal processing schemes, the above formulation holds, and further, the objective $g$ is a concave function (Section III-A). The central result of the present paper, which is described in detail in Section III, is that a PCFB is optimal for all such problems where $g$ is concave. The main reason for this is that whenever a PCFB exists, the search space $\mathcal{S}$ has a very special structure; its convex hull is a polytope (Section III-A). Since the set $\mathcal{S}$ plays an important role in the further discussion, we summarize the main definitions and facts pertaining to it.

\section{A. Summary of Definitions and Facts Related to the Search Space}

1) Definition: For each FB in the given class $\mathcal{C}$, the subband variance vector associated with the input process $x(n)$ is defined as the vector $\mathbf{v}=\left(\sigma_{0}^{2}, \sigma_{1}^{2}, \cdots, \sigma_{M-1}^{2}\right)^{T}$, where $\sigma_{i}^{2}$ is the variance of the process $v_{i}^{(x)}(n)$. Here, $v_{i}^{(x)}(n)$ is the $i$ th subband signal produced by feeding $x(n)$ as the FB input.

2) Computing the subband variance vector: Given the FB analysis polyphase matrix $\mathbf{E}(z)$ and the psd matrix $\mathbf{S}_{\mathbf{x x}}\left(e^{j \omega}\right)$ of the vector input $\mathbf{x}(n)$ in Fig. 1, the vector process $\left(v_{0}^{(x)}(n), v_{1}^{(x)}(n), \cdots, v_{M-1}^{(x)}(n)\right)^{T}$ has psd ma- 
trix $\mathbf{E}\left(e^{j \omega}\right) \mathbf{S}_{\mathbf{x x}}\left(e^{j \omega}\right) \mathbf{E}^{\dagger}\left(e^{j \omega}\right)$. Thus, the subband variance vector is

$$
\mathbf{v}=\frac{1}{2 \pi} \int_{0}^{2 \pi} \operatorname{diag}\left(\mathbf{E}\left(e^{j \omega}\right) \mathbf{S}_{\mathbf{x x}}\left(e^{j \omega}\right) \mathbf{E}^{\dagger}\left(e^{j \omega}\right)\right) d \omega
$$

3) The optimization search space is defined as the set $\mathcal{S}$ of all subband variance vectors corresponding to all FBs in the given class $\mathcal{C}$. Therefore, $\mathcal{S}$ is fully specified, given the class $\mathcal{C}$ and the input statistics $\mathbf{S}_{\mathbf{x x}}\left(e^{j \omega}\right)$. All entries of any vector in $\mathcal{S}$ are clearly non-negative. Thus, $\mathcal{S} \subset$ $\mathcal{R}_{+}^{M} \subset \mathcal{R}^{M}$.

4) The set $\mathcal{S}$ is bounded and lies entirely on an $M-1$ dimensional hyperplane in $\mathcal{R}^{M}$. This follows from (5), using the fact that $\mathbf{E}\left(e^{j \omega}\right)$ is unitary for all $\omega$ (orthonormality of the FB). No matter what the class $\mathcal{C}$, there is always an upper bound [depending only on $\mathbf{S}_{\mathbf{x x}}\left(e^{j \omega}\right)$ ] on all entries of all vectors $\mathbf{v} \in \mathcal{S}$. Thus, $\mathcal{S}$ is bounded. Also, the sum of the entries of $\mathbf{v}$ is the same for all $\mathbf{v} \in \mathcal{S}$, i.e., it is the trace of the matrix $(1 / 2 \pi) \int_{0}^{2 \pi} \mathbf{S}_{\mathrm{xx}}\left(e^{j \omega}\right) d \omega$. So $\mathcal{S}$ lies on an $M-1$ dimensional hyperplane in $\mathcal{R}^{M}$.

5) Permutation symmetry of $\mathcal{S}$. An FB is defined by an ordered set of analysis and synthesis filters. Therefore, a change of this ordering (or equivalently, interchanging of rows of the analysis polyphase matrix) technically produces a different FB, which we will refer to as a permutation of the original FB. However, clearly, all permutations of a uniform FB are essentially the same, i.e., equally easy to implement. Therefore, we make the following very reasonable assumption on the given class $\mathcal{C}$ of FBs: Any permutation of any FB in $\mathcal{C}$ is also in $\mathcal{C}$. This assumption holds for all specific classes $\mathcal{C}$ that we will encounter. Note that if two FBs are permutations of each other, then so are their subband variance vectors; however, the minimization objective may attain different values at these vectors. Thus, we use the convention of defining an FB as an ordered set of filter pairs because the ordering affects the objective.

\section{OPTIMALITY OF PCFBs}

We now show that PCFBs are optimal whenever the objective function to be minimized is concave on the optimization search space $\mathcal{S}$. The proof follows from strong connections between the notion of a PCFB and certain results in convexity and majorization theory reviewed in Section III-A. PCFBs are defined and described in Section III-B. In Section III-C, we show the connection between PCFBs and special convex sets called polytopes and thereby prove the main result of the paper.

\section{A. Convexity Theory [21]}

Convex Sets: A set $D \subset \mathcal{R}^{M}$ is defined to be convex if $\mathbf{x}, \mathbf{y} \in D$ implies $\mu \mathbf{x}+(1-\mu) \mathbf{y} \in D$ whenever $0 \leq \mu \leq 1$. Geometrically, $D$ is convex if any line segment with endpoints in $D$ lies wholly in $D$; see Fig. 2 . A convex combination of a finite set of vectors $\mathrm{x}_{i}, i=1,2, \cdots, N$ is by definition a vector of the form $\sum_{i=1}^{N} \alpha_{i} \mathbf{x}_{i}$ with $0 \leq \alpha_{i} \leq 1$ and $\sum_{i=1}^{N} \alpha_{i}=1$. Thus, by definition, $D$ is convex if any convex combination of

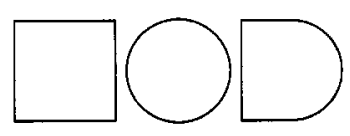

(a)

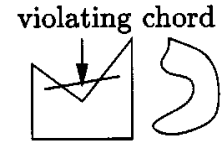

(b)

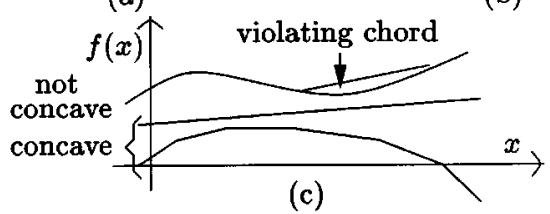

Fig. 2. Convex sets and concave functions. (a) Convex sets. (b) Nonconvex sets. (c) Concave functions of one variable.

any pair (or equivalently, by induction, any finite set) of elements of $D$ lies in $D$ [8], [23].

Concave Functions: Let $f$ be a real-valued function defined on a convex set $D \subset \mathcal{R}^{M}$. The function $f$ is defined to be concave on the domain $D$ if given any elements $\mathbf{x}, \mathbf{y}$ in $D$,

$$
\begin{aligned}
& f(\mu \mathrm{x}+(1-\mu) \mathbf{y}) \\
& \quad \geq \mu f(\mathbf{x})+(1-\mu) f(\mathbf{y}) \quad \text { whenever } 0 \leq \mu \leq 1 .
\end{aligned}
$$

Graphically, this means that the function $f$ is always above its chord; see Fig. 2(c). The domain $D$ of $f$ has to be convex to ensure that the argument of $f$ on the left side of (6) is in $D$, i.e., to ensure that the above definition makes sense. For a concave function $f$, we can use (6) to show by induction that for any $\mathbf{x}_{i} \in D$

$$
\begin{gathered}
f\left(\sum_{i=1}^{N} \alpha_{i} \mathbf{x}_{i}\right) \geq \sum_{i=1}^{N} \alpha_{i} f\left(\mathbf{x}_{i}\right) \quad \text { whenever } 0 \leq \alpha_{i} \leq 1 \\
\text { and } \sum_{i=1}^{N} \alpha_{i}=1 .
\end{gathered}
$$

This is known as Jensen's inequality. The function $f$ is said to be strictly concave if it is concave and further if equality in (6) is achieved for distinct $\mathbf{x}, \mathbf{y}$ iff $\mu$ is either 0 or 1 . For such $f$, equality is achieved in (7) for distinct $\mathbf{x}_{i}$ iff one of the $\alpha_{i}$ is unity (and, hence, all the others are zero).

Convex Hulls: The convex hull of a set $D \subset \mathcal{R}^{M}$ is denoted by $\operatorname{co}(D)$ and is defined as the set of all possible convex combinations of elements of $D$. Equivalently, it can be defined as the "smallest" (i.e., minimal) convex set containing $D$ or the intersection of all convex sets containing $D$. Thus, $D=\operatorname{co}(D)$ iff $D$ is a convex set.

Polytopes: A convex polytope is defined as the convex hull of a finite set. If $E \subset \mathcal{R}^{M}$ is finite, $P \triangleq \operatorname{co}(E)$ is a polytope. We can assume that no vector in $E$ is a convex combination of other vectors of $E$, as deleting such vectors from $E$ does not change $P$. With this condition, the polytope $P$ is said to be generated by the elements of $E$, and these elements are called the extreme points (or vertices or corners) of $P$; see Fig. 3(a)-(c). The following result on extreme points, which is illustrated by Fig. 3(d), is vital in explaining PCFB optimality.

Theorem 1-Optimality of Extreme Points of Polytopes: Let a function $f$ have a convex polytope $P$ as domain. If $f$ is concave on $P$, at least one extreme point of $P$ achieves the min- 


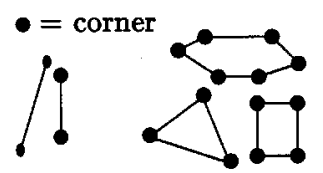

(a)

(b)

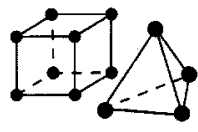

(c)

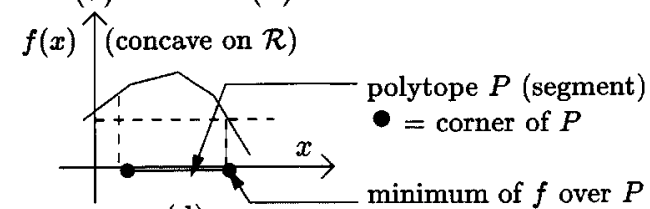

(d)
Fig. 3. $\quad M$-dimensional polytopes, their extreme points, and their optimality. (a) $M=1$. (b) $M=2$. (c) $M=3$. (d) Optimality of extreme points.

imum of $f$ over $P$. Further, if $f$ is strictly concave, its minimum over $P$ is necessarily at an extreme point of $P$.

Proof: Let $E$ be the set of extreme points of $P$. Thus, $E$ is finite, and $P=\operatorname{co}(E)$. Let $E=\left\{\mathbf{v}_{1}, \mathbf{v}_{2}, \cdots, \mathbf{v}_{N}\right\}$, and let $\mathbf{v}_{j} \in E$ attain the minimum of $f$ over the finite set $E$. Now, by definition of a polytope, for any $\mathbf{v} \in P$, we have $\mathbf{v}=\sum_{i=1}^{N} \alpha_{i} \mathbf{v}_{i}$ for some $\alpha_{i}$ such that $0 \leq \alpha_{i} \leq 1$ and $\sum_{i=1}^{N} \alpha_{i}=1$. Thus

$$
\begin{aligned}
f(\mathbf{v})= & f\left(\sum_{i=1}^{N} \alpha_{i} \mathbf{v}_{i}\right) \geq \sum_{i=1}^{N} \alpha_{i} f\left(\mathbf{v}_{i}\right) \\
& \quad \text { by (7), i.e., Jensen's inequality }] \\
\geq & \sum_{i=1}^{N} \alpha_{i} f\left(\mathbf{v}_{j}\right)=f\left(\mathbf{v}_{j}\right) \\
& \quad\left(\text { by definition of } \mathbf{v}_{j} \text { and using } \sum_{i=1}^{N} \alpha_{i}=1\right) .
\end{aligned}
$$

Thus, $f(\mathbf{v}) \geq f\left(\mathbf{v}_{j}\right)$, i.e., the extreme point $\mathbf{v}_{j}$ of $P$ attains the minimum of $f$ over $P$. Further, the $\mathbf{v}_{i}$ are distinct; therefore, if $f$ is strictly concave, then Jensen's inequality becomes strict unless one of the $\alpha_{i}$ is unity. Thus, in this case, the minimum is necessarily at an extreme point of $P$.

$\nabla \nabla \nabla$

Extreme Points of General Convex Sets: A point $\mathbf{v}$ in a convex set $D$ is said to be an extreme point of $D$ if it cannot be expressed as a nontrivial convex combination of points of $D$, i.e., $\mathbf{v}=\sum_{i=1}^{J} \alpha_{i} \mathbf{x}_{i}$ for $\mathbf{x}_{i} \in D$ and $0<\alpha_{i}<1, \sum_{i=1}^{J} \alpha_{i}=1$ implies $\mathrm{x}_{1}=\cdots=\mathrm{x}_{J}(=\mathbf{v})$. This definition can be verified to be equivalent to the earlier definition of extreme points of polytopes when $D$ is a polytope. Thus, a polytope is simply a convex set with finitely many extreme points. We may note (although we do not use) the fact that Theorem 1 holds even if the domain $P$ is a general compact convex set. This is proved in a very similar manner, using one additional key result: Every compact convex set is the convex hull of the set of its extreme points (Krein-Milman theorem) [21]. Polytopes are special compact convex sets (i.e., those with finitely many extreme points). Another easily proved fact on extreme points that we will use in Section III-C.3 is as follows: For any set $D$, the extreme points of $\operatorname{co}(D)$ always lie in $D$.

\section{B. PCFBs and Majorization: Definitions and Properties}

Definition-Majorization: Let $A=\left\{a_{0}, a_{1}, \cdots, a_{M-1}\right\}$ and $B=\left\{b_{0}, b_{1}, \cdots, b_{M-1}\right\}$ be two sets each having $M$ real numbers (not necessarily distinct). The set $A$ is defined to $m a$ jorize the set $B$ if the elements of these sets, when ordered so that $a_{0} \geq a_{1} \geq \cdots \geq a_{M-1}$ and $b_{0} \geq b_{1} \geq \cdots \geq b_{M-1}$, obey the property that

$$
\sum_{i=0}^{P} a_{i} \geq \sum_{i=0}^{P} b_{i} \text { for all } P=0,1, \cdots, M-1
$$$$
\text { with equality holding when } P=M-1 \text {. }
$$

Given two vectors $\mathbf{v}_{1}, \mathbf{v}_{2}$ in $\mathcal{R}^{M}$, we will say that $\mathbf{v}_{1}$ majorizes $\mathbf{v}_{2}$ when the set of entries of $\mathbf{v}_{1}$ majorizes that of $\mathbf{v}_{2}$. Evidently, in this case, any permutation of $\mathbf{v}_{1}$ majorizes any permutation of $\mathbf{v}_{2}$.

Definition-PCFBs: Let $\mathcal{C}$ be the given class of orthonormal uniform $M$-channel FBs, and let $\mathbf{S}_{\mathbf{x x}}\left(e^{j \omega}\right)$ be the power-spectrum matrix of the vector process input $\mathrm{x}(n)$ (shown in Fig. 1). An FB in $\mathcal{C}$ is said to be a PCFB for the class $\mathcal{C}$ for the input psd $\mathbf{S}_{\mathbf{x x}}\left(e^{j \omega}\right)$, if its subband variance vector (which is defined in Section II-A) majorizes the subband variance vector of every FB in the class $\mathcal{C}$.

Remarks on the PCFB Definition:

1) A Simple Optimality Property: In Fig. 1, suppose the FB has subbands numbered in decreasing order of their variances $\sigma_{i}^{2}$, i.e., $\sigma_{0}^{2} \geq \sigma_{1}^{2} \geq \cdots \geq \sigma_{M-1}^{2}$, and the $P_{i}$ are constant multipliers $m_{i}$ given by

$$
m_{i}= \begin{cases}1, & \text { for } 0 \leq i \leq P-1 \\ 0, & \text { for } P \leq i \leq M-1\end{cases}
$$

for a fixed integer $P$ with $0 \leq P \leq M$. This system keeps the $P$ strongest (largest variance) subbands and discards the others. If the desired output signal $d(n)$ equals the input $x(n)$, then all assumptions of Section II are satisfied, and the minimization objective indeed has the form of (4). The optimum FB is the one minimizing $(1 / M) \sum_{i=P}^{M-1} \sigma_{i}^{2}$. Now, all FBs have the same value of $\sum_{i=0}^{M-1} \sigma_{i}^{2}$; therefore, the optimum FB is the one maximizing $\sum_{i=0}^{P-1} \sigma_{i}^{2}$. Thus, from the definitions of PCFBs and majorization, it follows that a PCFB, if it exists, has the property of being optimum for this problem for all values of $P$. In fact, this property is the origin of the concept of a PCFB [24] and is clearly equivalent to its definition. PCFBs are also optimal for many other problems, as Section III-C will show.

2) Existence of $P C F B$ : Given the class $\mathcal{C}$ of $F B s$ and the input power spectrum $\mathbf{S}_{\mathbf{x x}}\left(e^{j \omega}\right)$, a PCFB for $\mathcal{C}$ may not always exist. The PCFB and its existence depends on both $\mathcal{C}$ and $\mathbf{S}_{\mathbf{x x}}\left(e^{j \omega}\right)$. For example, for white input $\left(\mathbf{S}_{\mathbf{x x}}\left(e^{j \omega}\right)=\right.$ identity matrix), all FBs in $\mathcal{C}$ are PCFBs, no matter what $\mathcal{C}$ is. Section IV studies certain classes $\mathcal{C}$ for which PCFBs always exist for any input psd $\mathbf{S}_{\mathbf{x x}}\left(e^{j \omega}\right)$ [of course, the 
PCFB will depend on $\mathbf{S}_{\mathbf{x x}}\left(e^{j \omega}\right)$ ]. Section V studies certain classes $\mathcal{C}$ for which PCFBs do not exist for large families of input spectra. ${ }^{2}$

3) Nonuniqueness of PCFB: From the definition of majorization, any permutation of a PCFB is also a PCFB. Further, it is possible that two FBs that are not permutations of each other are both PCFBs, i.e., the PCFB need not be unique. However, all PCFBs must have the same subband variance vector up to permutation. This is because two sets majorizing each other must be identical-a direct consequence of the definition of majorization. As all our FB optimizations involve not the actual FB but only its subband variance vector, we often speak of the PCFB, even though it may not be unique.

\section{Principal Components, Convex Polytopes, and PCFB Optimality}

Let $\mathcal{C}$ be the given class of orthonormal uniform $M$-channel FBs, and $\mathbf{S}_{\mathbf{x x}}\left(e^{j \omega}\right)$ the psd matrix of the vector input $\mathbf{x}(n)$ of Fig. 1. Let $\mathcal{S}$ be the set of all subband variance vectors of all FBs in $\mathcal{C}$ for input $\mathrm{x}(n)$. We have the following theorem.

Theorem 2-PCFBs and Convex Polytopes: A PCFB for the class $\mathcal{C}$ for input psd $\mathbf{S}_{\mathbf{x x}}\left(e^{j \omega}\right)$ exists if and only if the convex hull $\operatorname{co}(\mathcal{S})$ is a polytope whose extreme points consist of all permutations of a single vector $\mathbf{v}_{*}$. Under this condition, $\mathbf{v}_{*}$ is the subband variance vector produced by the PCFB.

Theorem 3-Optimality of PCFBs: The PCFB for the class $\mathcal{C}$ (if it exists) is the optimum $\mathrm{FB}$ in $\mathcal{C}$ whenever the minimization objective is a concave function on the domain $\operatorname{co}(\mathcal{S})$. Further if this function is strictly concave, the optimum FB is necessarily a PCFB.

Theorem 3 follows directly from Theorem 2 (which is proved in Section III-C-3) and Theorem 1 of Section III-A. Note that the FB optimization involves choosing the best vector from $\mathcal{S}$, but Theorem 1 is used here to find the best vector from $\operatorname{co}(\mathcal{S}) \supset \mathcal{S}$. However, Theorem 2 shows that the best vector from $\operatorname{co}(\mathcal{S})$ in fact lies in $\mathcal{S}$ (and corresponds to the PCFB). Hence, it must be optimum over $\mathcal{S}$. Note that all permutations of a PCFB are PCFBs, and the above theorems do not specify which of these is the optimum. All of them need not be equally good in general. However, the optimum can be found by a finite search over these PCFBs.

Theorem 3 shows optimality of PCFBs for a number of signal processing problems. In Section II, we had a general formulation of the FB optimization problem such that the minimization objective $g$ was purely a function of the subband variance vector, as in (4). If the functions $h_{i}$ in (4) are all concave on $\mathcal{R}_{+}$, then $g$ is concave on the domain $\operatorname{co}(\mathcal{S})$ [23]. This happens in several problems, as we will see in later sections. Thus, Theorem 3 shows PCFB optimality for all these problems. To prove Theorem 2 and, hence, Theorem 3, we first review some results on majorization theory [11].

1) Relevant Definitions from Majorization Theory:

a) A doubly stochastic matrix $\mathbf{Q}$ is a square matrix with nonnegative real entries $q_{i j}$ satisfying $\sum_{i} q_{i j}=1, \sum_{j} q_{i j}=$

${ }^{2} \mathrm{~A}$ question of possible interest is as follows: Given a class $\mathcal{C}$, find all nonwhite input spectra for which a PCFB for $\mathcal{C}$ exists.
1, i.e., the sum of the entries in any row or column of $\mathbf{Q}$ is unity. All convex combinations and products of $M \times$ $M$ doubly stochastic matrices are also doubly stochastic (Appendix A).

b) Permutation matrices are square matrices obtained by permuting rows (or columns) of the identity matrix. Thus, they are doubly stochastic. In fact, they are the only unitary doubly stochastic matrices. (This is because $\sum_{i=1}^{M} p_{i}=\sum_{i=1}^{M} p_{i}^{2}=1$ for non-negative $p_{i}$ iff all but one of the $p_{i}$ are zero.)

c) An orthostochastic matrix $\mathbf{Q}$ is one that can be obtained from a unitary matrix $\mathbf{U}$ by replacing each element $u_{i j}$ by $q_{i j}=\left|u_{i j}\right|^{2}$. We will refer to $\mathbf{Q}$ as the orthostochastic matrix corresponding to the unitary matrix $\mathbf{U}$. Since $\sum_{i}\left|u_{i j}\right|^{2}=\sum_{j}\left|u_{i j}\right|^{2}=1$ for unitary $\mathbf{U}$, every $M \times M$ orthostochastic matrix is doubly stochastic. The converse is true if $M \leq 2$ but is false if $M>2$ (see Appendix B).

2) Relevant Results from Majorization Theory:

i) Majorization Theorem [10], [11]: If $\mathbf{a}, \mathbf{b} \in \mathcal{R}^{M}$, a majorizes $\mathbf{b}$ iff $\mathbf{b}=\mathbf{Q} \mathbf{a}$ for some doubly stochastic $\mathbf{Q}$.

ii) Birkhoff's Theorem [11]: A matrix $\mathbf{Q}$ is doubly stochastic if and only if it is a convex combination of finitely many permutation matrices, i.e., there are finitely many permutation matrices $\mathbf{P}_{i}$ such that

$$
\sum_{i=1}^{N} \alpha_{i} \mathbf{P}_{i}=\mathbf{Q}, \quad \text { where } 0 \leq \alpha_{i} \leq 1, \quad \text { and } \sum_{i=1}^{N} \alpha_{i}=1 .
$$

iii) Orthostochastic Majorization Theorem [11]: For $\mathbf{a}, \mathbf{b} \in$ $\mathcal{R}^{M}$, the following statements are equivalent.

a) $a$ majorizes $\mathrm{b}$.

b) There exists an orthostochastic matrix $\mathbf{Q}$ (corresponding to a unitary matrix $\mathbf{U}$ ) such that $\mathbf{b}=\mathbf{Q a}$.

c) There is a Hermitian matrix $\mathbf{H}$ with entries of $\mathbf{a}$ as its eigenvalues and entries of $\mathbf{b}$ on its diagonal.

On the Proofs: The majorization theorem actually follows from the orthostochastic majorization theorem (see [10] or [29] for an independent proof). Regarding Birkhoff's theorem, as all permutation matrices are doubly stochastic, so is their convex combination $\mathbf{Q}$ of (12) (see Appendix A). The converse proof is more elaborate [11]. In the orthostochastic majorization theorem, equivalence of b) and c) is easily proved. The key idea is that for any diagonal matrix $\boldsymbol{\Lambda}$ and unitary matrix $\mathbf{T}$, $\operatorname{diag}\left(\mathbf{T} \boldsymbol{\Lambda} \mathbf{T}^{\dagger}\right)=\mathbf{Q} \operatorname{diag}(\boldsymbol{\Lambda})$, where $\mathbf{Q}$ is the orthostochastic matrix corresponding to $\mathbf{T}$. This is because if $t_{i j}$ is the $i j$ th entry of $\mathbf{T}$ and $\operatorname{diag}(\boldsymbol{\Lambda})=\left(\lambda_{0}, \lambda_{1}, \cdots, \lambda_{M-1}\right)^{T}$, the $i$ th diagonal entry of $\mathbf{T} \boldsymbol{\Lambda} \mathbf{T}^{\dagger}$ is $\sum_{j=0}^{M-1}\left|t_{i j}\right|^{2} \lambda_{j}$, which is exactly the $i$ th entry of $\mathbf{Q d i a g}(\boldsymbol{\Lambda})$. Therefore, given $\mathrm{b}$ ), we choose $\operatorname{diag}(\boldsymbol{\Lambda})=\mathbf{a}$ and prove c) by setting $\mathbf{H}=\mathbf{U} \boldsymbol{\Lambda} \mathbf{U}^{\dagger}$. Conversely, given c), we prove b) by letting $\mathbf{U}$ be a unitary matrix diagonalizing $\mathbf{H}$, i.e., satisfying $\mathbf{H}=\mathbf{U} \boldsymbol{\Lambda} \mathbf{U}^{\dagger}$ for diagonal $\boldsymbol{\Lambda}$.

That b) [or c)] implies a) follows from the majorization theorem since all $M \times M$ orthostochastic matrices are doubly stochastic. As the converse is false unless $M \leq 2$ (see Appendix B), the result that a) implies that b) [or c)] is stronger than the corresponding result in the "plain" majorization theorem. This result is not used until Section IV-B. Its proof is more involved 
[11]. The fact that c) implies a) is in fact precisely the statement that the KLT is the PCFB for the class of transform coders, as elaborated in Section IV-B.

Proof of Theorem 2: Let a PCFB for the class $\mathcal{C}$ exist for the given input psd $\mathbf{S}_{\mathbf{x x}}\left(e^{j \omega}\right)$. Let $\mathbf{v}_{*}$ be the PCFB subband variance vector (unique up to permutation; see Section III-B). Let $\mathbf{P}_{j}$ be the $M \times M$ permutation matrices for $j=1,2, \cdots, J$ (where $J=M$ !), and let $\mathbf{v}_{j} \triangleq \mathbf{P}_{j} \mathbf{v}_{*}$. Thus, $E \triangleq\left\{\mathbf{v}_{j}: j=\right.$ $1,2, \cdots, J\}$ is the (finite) set of all permutations of $\mathbf{v}_{*}$. We have to prove that $\operatorname{co}(\mathcal{S})=\operatorname{co}(E)$. For this, take any $\mathbf{v} \in$ $\mathcal{S}$. By definition of PCFBs, $\mathbf{v}_{*}$ majorizes $\mathbf{v}$. Therefore, by the majorization theorem (Section III-C1), $\mathbf{v}=\mathbf{Q} \mathbf{v}_{*}$ for some doubly stochastic matrix $\mathbf{Q}$. By Birkhoff's theorem (see Section III-C.1), $\mathbf{Q}$ is some convex combination of the $\mathbf{P}_{j}$. Thus

$$
\begin{gathered}
\mathbf{v}=\mathbf{Q} \mathbf{v}_{*}=\sum_{j=1}^{J} \alpha_{j} \mathbf{P}_{j} \mathbf{v}_{*}=\sum_{j=1}^{J} \alpha_{j} \mathbf{v}_{j} \text { for some } \alpha_{j} \\
\text { such that } 0 \leq \alpha_{j} \leq 1, \sum_{j=1}^{J} \alpha_{j}=1 .
\end{gathered}
$$

Therefore, every $\mathbf{v} \in \mathcal{S}$ is a convex combination of the $\mathbf{v}_{j}$, i.e., $\mathcal{S} \subseteq \operatorname{co}(E)$; hence, $\operatorname{co}(\mathcal{S}) \subseteq \operatorname{co}(\operatorname{co}(E))=\operatorname{co}(E)$. However, by permutation-symmetry of $\mathcal{S}$ (Section II-A), $E \subset \mathcal{S}$, and therefore, $\operatorname{co}(E) \subseteq \operatorname{co}(\mathcal{S})$. Combining, $\operatorname{co}(\mathcal{S})=\operatorname{co}(E)$, as desired.

Conversely, let $\mathbf{v}_{*}$ be a vector such that with $\mathbf{v}_{j}=\mathbf{P}_{j} \mathbf{v}_{*}$ and $E \triangleq\left\{\mathbf{v}_{j}: j=1,2, \cdots, J\right\}$, we have $\operatorname{co}(\mathcal{S})=\operatorname{co}(E)$. We then have to prove that a PCFB for the class $\mathcal{C}$ exists for the given input psd and that $\mathbf{v}_{*}$ is a PCFB subband variance vector. To do this, note that $\mathcal{S} \subseteq \operatorname{co}(\mathcal{S})=\operatorname{co}(E)$. Thus, if $\mathbf{v} \in \mathcal{S}$, then $\mathbf{v} \in \operatorname{co}(E)$ so that $\mathbf{v}$ can be written as a convex combination of the elements $\mathbf{v}_{j} \in E$. Therefore, there are $\alpha_{j}$ such that

$$
0 \leq \alpha_{j} \leq 1, \quad \sum_{j=1}^{J} \alpha_{j}=1
$$

and

$$
\begin{gathered}
\mathbf{v}=\sum_{j=1}^{J} \alpha_{j} \mathbf{v}_{j}=\sum_{j=1}^{J} \alpha_{j} \mathbf{P}_{j} \mathbf{v}_{*}=\mathbf{Q} \mathbf{v}_{*} \\
\text { where } \mathbf{Q} \triangleq \sum_{j=1}^{J} \alpha_{j} \mathbf{P}_{j} .
\end{gathered}
$$

Here, $\mathbf{Q}$ is a convex combination of permutation matrices $\mathbf{P}_{j}$; therefore, it is doubly stochastic (Birkhoff's theorem). As $\mathbf{v}=$ $\mathbf{Q} \mathbf{v}_{*}$, by the majorization theorem, $\mathbf{v}_{*}$ majorizes $\mathbf{v}$. Thus, an FB with subband variance vector $\mathbf{v}_{*}$ will be a PCFB for the given class $\mathcal{C}$ and input psd. Indeed, there is such an FB in $\mathcal{C}$ : As $\operatorname{co}(\mathcal{S})=\operatorname{co}(E)$ and $E$ is a finite set, the extreme points of the polytope $\operatorname{co}(\mathcal{S})$ lie within $E$, and they also lie in $\mathcal{S}$ (Section III-A). Thus, $\mathbf{v}_{j} \in \mathcal{S}$ for at least one $j$ and, hence, for all $j$ (by the permutation-symmetry of $\mathcal{S}$; see Section II-A). $\quad \nabla \nabla \nabla$

Note that in general, all we can say about the extreme points of a polytope $\operatorname{co}(E)$ is that they lie in $E$. Here, however, with $E$ as the (finite) set of all permutations of $\mathbf{v}_{*}$, in fact all points in $E$ are extreme points of $\operatorname{co}(E)$, i.e., no vector in $E$ is expressible as a convex combination of other vectors of $E$. This is provable by induction on the vector dimension $M$. Let $\mathbf{v}_{1}=\sum_{j=2}^{j} \alpha_{j} \mathbf{v}_{j}$ with $0 \leq \alpha_{j} \leq 1$ and $\sum_{j=2}^{J} \alpha_{j}=1$. Then, the greatest entry of $\mathbf{v}_{1}$ is a convex combination of real numbers no greater than itself. Therefore, all these numbers must be equal. Deleting from each $\mathbf{v}_{j}$ the entry corresponding to this number yields the induction hypothesis.

Functions Minimized by Majorization: Currently known instances of PCFB optimality in signal processing problems arise from minimization objectives of the form (4), where the functions $h_{i}$ are concave on $\mathcal{R}_{+}$. Theorem 3 , of course, shows PCFB optimality for a more general family of objectives, namely, those that are concave in the subband variance vector [and need not necessarily have the special form of (4)]. In fact, even this is not the complete family of objectives minimized by PCFBs. For example, if $g$ is a monotone increasing function on $\mathcal{R}$, then for any concave objective $\phi(\cdot)$, clearly, $g(\phi(\cdot))$ is also minimized by PCFBs. Unless $g$ is also concave, in general, this new function is not concave. A specific nonconcave example of this kind is generated by $\phi\left(x_{1}, \cdots, x_{M}\right)=\sum_{i} \log \left(x_{i}\right)$ and $g(y)=e^{y}$, giving $g(\phi(\cdot))=\psi\left(x_{1}, \cdots, x_{M}\right)=\prod_{i} x_{i}$.

If attention is restricted to symmetric functions [i.e., functions $\phi$ obeying $\phi(\mathbf{P x})=\phi(\mathbf{x})$ for all $\mathbf{x}$ if $\mathbf{P}$ is any permutation matrix], then the functions minimized by majorization are said to be Schur-concave [18]. To be precise, $\phi$ is said to be Schur-concave if $\phi(\mathbf{x}) \leq \phi(\mathbf{y})$ whenever $\mathbf{x}$ majorizes $\mathbf{y}$. (This implies symmetry of $\phi$ since Px majorizes $\mathbf{x}$ for any permutation matrix P.) Thus, symmetric concave functions are examples of Schur-concave functions, whereas the function $\psi$ defined earlier is a Schur-concave function that is not concave. Clearly, PCFBs minimize all Schur-concave objectives. Full characterizations and several interesting examples of such functions can be found in [18].

\section{PCFBS FOR STANDARD CLASSES AND OPTIMALITY FOR COMPRESSION}

This section first shows existence of PCFBs for three special classes of FBs, namely, classes with $M=2$ channels, the class of $M$-channel orthogonal transform coders, and that of all $M$-channel orthonormal FBs. This well-known result is reviewed to show how it fits in the framework of the earlier sections, which have not yet been restricted to any specific class of FBs. We also prove the convexity of the search-space for these classes, which has not been observed earlier. We then review PCFB optimality for data compression.

To begin, let $\mathcal{C}$ be any class of uniform orthonormal two channel FBs, e.g., that of FIR or IIR FBs with a given bound on the filter order. Irrespective of the input psd matrix, all realizable subband variance vectors $\left(\sigma_{0}^{2}, \sigma_{1}^{2}\right)^{T}$ in the search-space $\mathcal{S} \subset \mathcal{R}^{2}$ then have the same value of $\sigma_{0}^{2}+\sigma_{1}^{2}$ (Section II-A). Thus, $\mathcal{S}$ lies wholly on a line of slope -1 in $\mathcal{R}^{2}$. Therefore, $\operatorname{co}(\mathcal{S})$ is an interval on this line; see Fig. 4. Thus, $\operatorname{co}(\mathcal{S})$ is a polytope with two extreme points, namely, the endpoints of the interval. By the definition, a PCFB is simply an FB maximizing one subband variance, thereby minimizing the other. Therefore, 


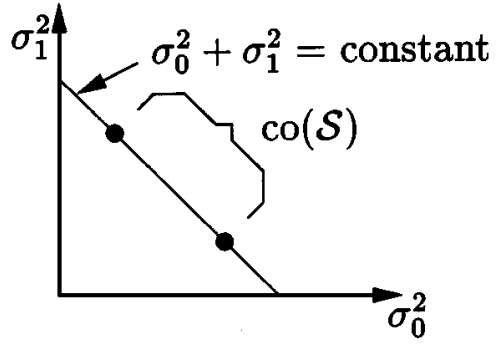

Fig. 4. Search space $\mathcal{S}$ for a class of two-channel FBs.

it always exists for such classes $\mathcal{C}$ and corresponds to the two extreme points of $\operatorname{co}(\mathcal{S})$, irrespective of the input psd. ${ }^{3}$

\section{A. Transform Coder Class}

The transform coder class $\mathcal{C}^{t}$ is defined as the class of uniform $M$-channel orthonormal FBs whose polyphase matrix $[\mathbf{E}(z)$ in Fig. 1] is a constant unitary matrix $\mathbf{T}$. In effect, we can speak of $\mathcal{C}^{t}$ as being the set of all $M \times M$ unitary matrices. Let $\mathbf{R}_{\mathrm{xx}}$ be the autocorrelation matrix of the input $\mathrm{x}(n)$ of Fig. 1. We then have the following theorem.

Theorem 4-Transform Coders-KLT, PCFBs, and Polytopes:

1) A PCFB always exists for $\mathcal{C}^{t}$. Hence, the set $\mathcal{S}$ of realizable subband variance vectors for $\mathcal{C}^{t}$ has a convex hull $\operatorname{co}(\mathcal{S})$ that is a polytope, as stated by Theorem 2 in Section III-C.

2) A unitary matrix $\mathbf{T} \in \mathcal{C}^{t}$ is a PCFB for $\mathcal{C}^{t}$ iff it diagonalizes $\mathbf{R}_{\mathbf{x x}}$, i.e., $\mathbf{T} \mathbf{R}_{\mathbf{x x}} \mathbf{T}^{\dagger}$ is diagonal. In other words, $\mathbf{T}$ is a PCFB for $\mathcal{C}^{t}$ iff it is the Karhunen-Loeve transform (KLT) for the input, i.e., it decorrelates the input [the subband signals $v_{i}^{(x)}(n), i=0,1, \cdots, M-1$ are uncorrelated for each time instant $n$ ].

3) $\mathcal{S}=\operatorname{co}(\mathcal{S})$. Therefore, $\mathcal{S}$ itself is a polytope with extreme points as permutations of the KLT subband variance vector.

Proof: The subband variance vector computation (5) becomes

$$
\begin{aligned}
& \mathbf{v}=\operatorname{diag}\left(\mathbf{R}_{\mathbf{v v}}\right), \quad \text { where } \mathbf{R}_{\mathbf{v v}}=\mathbf{T R}_{\mathbf{x x}} \mathbf{T}^{\dagger} \\
& \text { where } \mathbf{R}_{\mathbf{x x}}=\frac{1}{2 \pi} \int_{0}^{2 \pi} \mathbf{S}_{\mathbf{x x}}\left(e^{j \omega}\right) d \omega .
\end{aligned}
$$

Here, $\mathbf{R}_{\mathbf{v v}}$ is the autocorrelation matrix of the vector process $\left(v_{0}^{(x)}(n), v_{1}^{(x)}(n), \cdots, v_{M-1}^{(x)}(n)\right)^{T}$ of Fig. 1 . The input KLT is defined as the FB with unitary polyphase matrix $\mathbf{K}$ that diagonalizes $\mathbf{R}_{\mathbf{x x}}$, i.e., such that $\mathbf{K R}_{\mathbf{x x}} \mathbf{K}^{\dagger}=\boldsymbol{\Lambda}$ is a diagonal matrix. Thus, $\mathbf{v}_{*} \triangleq \operatorname{diag}(\boldsymbol{\Lambda})$ is the subband variance vector of the KLT and has as entries the eigenvalues of $\mathbf{R}_{\mathbf{x x}}$. Now, the Hermitian matrix $\mathbf{R}_{\mathbf{v v}}=\mathbf{T R}_{\mathbf{x x}} \mathbf{T}^{\dagger}=\mathbf{T} \mathbf{K}^{\dagger} \boldsymbol{\Lambda} \mathbf{K} \mathbf{T}^{\dagger}$ has entries of $\mathbf{v}$ on its diagonal and entries of $\mathbf{v}_{*}$ as its eigenvalues. Hence, $\mathbf{v}_{*}$ majorizes $\mathbf{v}$ by the orthostochastic majorization theorem of Section III-C1 [specifically by the fact that c) implies a) in its statement]. This shows that the KLT is a PCFB, which

${ }^{3}$ If $\operatorname{co}(\mathcal{S})$ is an open interval (i.e., one not containing its endpoints), no single FB achieves the maximum subband variance; hence, there is no PCFB. However, this situation is contrived and does not happen for most natural FB classes and input psds. is a well-known result. Conversely, if $\mathbf{T}$ is a PCFB for $\mathcal{C}^{t}$, then $\mathbf{v}=\mathbf{v}_{*}$ (up to permutation). Therefore, the Hermitian matrix $\mathbf{R}_{\mathbf{v v}}$ has its eigenvalues as its diagonal elements and is hence necessarily diagonal, i.e., $\mathbf{T}$ is the KLT for the input.

Finally, to show that $\mathcal{S}$ is the polytope $\operatorname{co}(\mathcal{S})$, take any $\mathbf{v} \in$ $\operatorname{co}(\mathcal{S})$. Then, $\mathbf{v}_{*}$ majorizes $\mathbf{v}$. We now make a stronger application of the orthostochastic majorization theorem, i.e., that a) implies c) in its statement in Section III-C.1. This shows that there is a Hermitian matrix $\mathbf{R}_{\mathbf{v v}}$ with the entries of $\mathbf{v}_{*}$ as its eigenvalues and those of $\mathbf{v}$ on its diagonal. As $\mathbf{R}_{\mathbf{v v}}, \mathbf{R}_{\mathbf{x x}}$ have the same eigenvalues, they are "similar," i.e., $\mathbf{U} \mathbf{R}_{\mathbf{x x}} \mathbf{U}^{\dagger}=\mathbf{R}_{\mathbf{v v}}$ for some unitary matrix $\mathbf{U}$. Therefore, the FB $\mathbf{U} \in \mathcal{C}^{t}$ has subband variance vector $\operatorname{diag}\left(\mathbf{U R}_{\mathbf{x x}} \mathbf{U}^{\dagger}\right)=\operatorname{diag}\left(\mathbf{R}_{\mathbf{v v}}\right)=\mathbf{v}$. Thus, $\mathbf{v}$ is a realizable subband variance vector for $\mathcal{C}^{t}$, i.e., $\mathbf{v} \in \mathcal{S}$. This holds for any $\mathbf{v} \in \operatorname{co}(\mathcal{S})$, so that $\mathcal{S}=\operatorname{co}(\mathcal{S})$.

\section{B. Unconstrained Class}

The class $\mathcal{C}^{u}$ is defined to contain all uniform $M$-channel orthonormal FBs with no constraints on the filters besides those imposed by orthonormality. Therefore, FBs in $\mathcal{C}^{u}$ could have ideal unrealizable filters. We could in effect think of $\mathcal{C}^{u}$ as the set of all $M \times M$ matrices $\mathbf{E}\left(e^{j \omega}\right)$ that are unitary for all $\omega .\left[\mathbf{E}\left(e^{j \omega}\right)\right.$ represents the analysis polyphase matrix.] An exact analog of Theorem 4 holds for this class as well. The only difference is in the construction of the PCFB from the given input psd matrix $\mathbf{S}_{\mathbf{x x}}\left(e^{j \omega}\right)$, which was first described in [25] and [26]. This section reviews this construction and proves the result $\mathcal{S}=\operatorname{co}(\mathcal{S})$ for the class $\mathcal{C}^{u}$.

PCFB Construction: Let $\mathbf{K}\left(e^{j \omega}\right) \in \mathcal{C}^{u}$ diagonalize $\mathbf{S}_{\mathbf{x x}}\left(e^{j \omega}\right)$ for each $\omega$, i.e., $\mathbf{K}\left(e^{j \omega}\right) \mathbf{S}_{\mathbf{x x}}\left(e^{j \omega}\right) \mathbf{K}^{\dagger}\left(e^{j \omega}\right)=\boldsymbol{\Lambda}\left(e^{j \omega}\right)$, where $\boldsymbol{\Lambda}\left(e^{j \omega}\right)$ is diagonal (for all $\omega$ ), and $\operatorname{diag}\left(\boldsymbol{\Lambda}\left(e^{j \omega}\right)\right)=$ $\left(\lambda_{0}\left(e^{j \omega}\right), \lambda_{1}\left(e^{j \omega}\right), \cdots, \lambda_{M-1}\left(e^{j \omega}\right)\right)^{T}$. Using (5), the subband variance vector $\mathbf{v}$ of an arbitrary $\mathrm{FB} \mathbf{E}\left(e^{j \omega}\right) \in \mathcal{C}^{u}$ is given by

$$
\begin{gathered}
2 \pi \mathbf{v}=\int_{0}^{2 \pi} \operatorname{diag}\left(\mathbf{E}\left(e^{j \omega}\right) \mathbf{S}_{\mathbf{x x}}\left(e^{j \omega}\right) \mathbf{E}^{\dagger}\left(e^{j \omega}\right)\right) d \omega \\
=\int_{0}^{2 \pi} \mathbf{Q}\left(e^{j \omega}\right)\left(\lambda_{0}\left(e^{j \omega}\right), \lambda_{1}\left(e^{j \omega}\right),\right. \\
\left.\cdots, \lambda_{M-1}\left(e^{j \omega}\right)\right)^{T} d \omega .
\end{gathered}
$$

Here, at each $\omega, \mathbf{Q}\left(e^{j \omega}\right)$ is the orthostochastic matrix corresponding to the unitary matrix $\mathbf{E}\left(e^{j \omega}\right) \mathbf{K}^{\dagger}\left(e^{j \omega}\right)$. Therefore, at each frequency $\omega$, the integrand vector of (16) produced by the FB $\mathbf{K}\left(e^{j \omega}\right) \in \mathcal{C}^{u}$ majorizes the corresponding vector of any $\mathrm{FB}$ in $\mathcal{C}^{u}$. This holds no matter how we order the eigenvalues $\lambda_{i}\left(e^{j \omega}\right)$ in (16). The integration process preserves this majorization relation if and only if the $\lambda_{i}\left(e^{j \omega}\right)$ are "ordered consistently" at all $\omega$. By this, we mean that if we number the $\lambda_{i}\left(e^{j \omega}\right)$ so that the entries of $\mathbf{v}$ are in descending order, then $\lambda_{0}\left(e^{j \omega}\right) \geq \lambda_{1}\left(e^{j \omega}\right) \geq \cdots \geq \lambda_{M-1}\left(e^{j \omega}\right)$ for all $\omega$. Thus, an FB $K\left(e^{j \omega}\right) \in \mathcal{C}^{u}$ is a PCFB for $\mathcal{C}^{u}$ iff it causes two effects: 1) totally decorrelating the input, i.e., diagonalizing its psd matrix $\mathbf{S}_{\mathbf{x x}}\left(e^{j \omega}\right)$, and 2) causing spectral majorization [26], which is the said ordering of eigenvalues of $\mathbf{S}_{\mathbf{x x}}\left(e^{j \omega}\right)$. Note that the PCFB for $\mathcal{C}^{u}$ has uncorrelated subband processes, unlike the instantaneous decorrelation produced by the KLT (Theorem 4).

Proving $\mathcal{S}=\operatorname{co}(\mathcal{S})$ : To prove this property for the class $\mathcal{C}^{u}$, let $\mathbf{v}_{*}$ be the PCFB subband variance vector, and let $\mathbf{v} \in$ 
$\operatorname{co}(\mathcal{S})$. Then, $\mathbf{v}_{*}$ majorizes $\mathbf{v}$. Therefore, by the orthostochastic majorization theorem (Section III-C.1), $\mathbf{v}=\mathbf{Q} \mathbf{v}_{*}$ for some orthostochastic matrix $\mathbf{Q}$ corresponding to a unitary matrix $\mathbf{U}$. Thus, if $\mathbf{K}\left(e^{j \omega}\right)$ is the polyphase matrix of the PCFB for $\mathcal{C}^{u}$, (16) shows that the FB in $\mathcal{C}^{u}$ with polyphase matrix $\mathbf{U K}\left(e^{j \omega}\right)$ produces subband variance vector $\mathbf{v}$, i.e., $\mathbf{v} \in \mathcal{S}$. This shows $\mathcal{S}=\operatorname{co}(\mathcal{S})$.

\section{PCFB Optimality for Coding/Compression}

Here, we consider the problems of [14] and [26], where the processors $P_{i}$ of Fig. 1 are quantizers, and the desired output $d(n)$ equals the input $x(n)$. This situation fits the general problem formulation of Section II under appropriate quantizer models. The subband error signal $v_{i}^{(e)}(n)$ of Section II here represents the $i$ th subband quantization noise. Under the quantizer model, we assume that this noise is zero mean with variance

$$
E\left[\left|v_{i}^{(e)}(n)\right|^{2}\right]=f_{i}\left(b_{i}\right) \sigma_{i}^{2}
$$

Here, $b_{i}$ is the number of bits allocated to the $i$ th quantizer, and $f_{i}$ is a characteristic of the quantizer called the normalized quantizer function [14]. We assume that $f_{i}$ does not depend on the FB in any way and that the quantization noise processes in different subbands are jointly stationary. The problem then fits the formulation of Section II. Comparing (17) with (3) reveals the minimization objective $g$ to be as in (4), i.e.,

$$
\begin{aligned}
& g\left(\sigma_{0}^{2}, \sigma_{1}^{2}, \cdots, \sigma_{M-1}^{2}\right) \\
& \quad=\frac{1}{M} \sum_{i=0}^{M-1} h_{i}\left(\sigma_{i}^{2}\right) \quad \text { with } h_{i}(x)=f_{i}\left(b_{i}\right) x
\end{aligned}
$$

Thus, the $h_{i}$ are linear (and hence concave); therefore, $g$ is indeed concave. Therefore, by Theorem 3, the PCFB if it exists is optimal for this problem. This is true no matter what the bit allocation $b_{i}$ is.

It is important to note that for the validity of our assumptions of Section II (and hence for PCFBs to be optimal), the function $h_{i}(x)=f_{i}\left(b_{i}\right) x$ must not depend on the $F B$ in any way. This is often not the case. In quantizers optimized to their input probability density function (pdf), $f_{i}$ depends on the $i$ th subband pdf, which in turn is influenced by choice of FB. Even with the model of [26], i.e., uniform quantization under the high bit rate approximation, $f_{i}\left(b_{i}\right)=c_{i} 2^{-2 b_{i}}$, where the constant $c_{i}$ (and hence $f_{i}$ ) depends on the $i$ th subband pdf. If we further assume the input to be a Gaussian random process, then all subbands have Gaussian pdf independent of choice of FB. For this special case, all $c_{i}$ are equal and constant, and the PCFB is indeed optimal. The need for these assumptions is illustrated by Feng and Effros [9], who demonstrate that the KLT is not the optimal orthogonal transform if the input has a uniform distribution.

For the case when $f_{i}\left(b_{i}\right)=c 2^{-2 b_{i}}$ (for which the PCFB is optimal), the optimal bit allocation $b_{i}$ (subject to a constraint on the total bit budget $\sum_{i=0}^{M-1} b_{i}=B$ ) is explicitly computable using the arithmetic mean-geometric mean (AM-GM) inequality. The objective under this bit allocation becomes the GM of the subband variances, i.e., $g=\left(\prod_{i=0}^{M-1} \sigma_{i}^{2}\right)^{(1 / M)}$. Minimizing this is equivalent to minimizing $\log (g)=(1 / M) \sum_{i=0}^{M-1} \log \left(\sigma_{i}^{2}\right)$. This is a concave function of the subband variance vector because $\log (x)$ is concave in $x$. For general quantizer functions $f_{i}$, the optimizations of the FB and the bit allocation have been decoupled since the PCFB is optimum for all bit allocations [14]. However, note that different permutations of a PCFB may be optimal for different bit allocations. In addition, computing the optimum bit allocation may be more involved. We can, however, prove one intuitive statement about the optimum $b_{i}$ in the special case when all $f_{i}$ are equal to a decreasing function $f$. In this case, a subband with larger variance receives more bits.

In (18), all $h_{i}$ are linear, i.e., $h_{i}(x)=m_{i} x+c_{i}$ for constants $m_{i}, c_{i}\left(m_{i}=f_{i}\left(b_{i}\right), c_{i}=0\right)$. In such cases, we can algebraically prove PCFB optimality [14] without using any result on majorization. As $c_{i}$ are constants, the optimization is unaffected by taking $c_{i}=0$. With $m_{0} \leq m_{1} \leq \cdots \leq m_{M-1}$ and $\sigma_{0}^{2} \geq \sigma_{1}^{2} \geq \cdots \geq \sigma_{M-1}^{2}$

$$
\begin{aligned}
M g\left(\sigma_{0}^{2}, \sigma_{1}^{2}, \cdots, \sigma_{M-1}^{2}\right)= & \sum_{i=0}^{M-1} m_{i} \sigma_{i}^{2} \\
= & \sum_{i=0}^{M-2}\left(m_{i}-m_{i+1}\right)\left(\sum_{j=0}^{i} \sigma_{j}^{2}\right) \\
& +m_{M-1}\left(\sum_{j=0}^{M-1} \sigma_{j}^{2}\right) \cdot
\end{aligned}
$$

As the last term is constant for all FBs, and since $m_{i}-m_{i+1} \leq$ 0 , the above $g$ is minimized by the PCFB, which, by definition, maximizes all the partial sums $\sum_{j=0}^{i} \sigma_{j}^{2}$ for $i=0,1, \cdots, M-$ 2 . This proof shows two noteworthy facts not shown by the earlier proof: 1) It exhibits the best permutation of the PCFB to be used, namely, that in which the largest subband variance $\sigma_{i}^{2}$ is associated with the least $m_{i}$, and so on. 2) It shows that the optimum FB is necessarily a PCFB if the $m_{i}$ are distinct. However, this simple approach works only for linear $h_{i}$ and thus fails for many of the problems of Section VI that result in nonlinear concave $h_{i}$.

\section{Filterbank Classes Having No PCFB}

Existence of a PCFB for a class $\mathcal{C}$ of orthonormal FBs implies a very strong condition on the subband variance vectors of the FBs in $\mathcal{C}$. There are many classes $\mathcal{C}$ that do not have PCFBs. Indeed, it seems quite plausible that the classes of Section IV are the only ones having PCFBs for all input power spectra. This section reviews some known results on nonexistence of PCFBs and shows that the classes of ideal DFT and cosine-modulated FBs do not have PCFBs for several input spectra.

If a PCFB for the given class $\mathcal{C}$ of FBs exists, it simultaneously optimizes over $\mathcal{C}$ several functions of the subband variances (Section III). Therefore, we can show nonexistence of PCFBs for $\mathcal{C}$ by proving that no single $\mathrm{FB}$ in $\mathcal{C}$ can optimize two of such functions. This method is used in [15] and [19] for certain classes of FIR FBs for a fixed input psd. The two functions used are the largest subband variance and the coding gain, which are both maximized by a PCFB if it exists. However, all 
optimizations are numerical. Nonexistence of PCFBs has not yet been proved for any reasonably general FIR class, say, the class of all $M$-channel $(M>2)$ FIR orthonormal FBs with polyphase matrix of McMillan degree $\mu>0$ (although it seems very likely that such classes do not have PCFBs). We now prove nonexistence of PCFBs for the classes of DFT and cosine-modulated FBs.

Definition: The class $\mathcal{C}^{\text {dft }}$ of $M$-channel orthonormal $D F T$ $F B s$ is the one containing all FBs as in Fig. 1 where the analysis filters are related by $H_{k}\left(e^{j \omega}\right)=P\left(e^{j(\omega-(2 \pi k / M))}\right)$ for some filter $P\left(e^{j \omega}\right)$ called the prototype. For example, any $P\left(e^{j \omega}\right)$ that has an alias-free $(M)$ support and has constant magnitude on its support [and is thus Nyquist $(M)$ ] produces an FB in $\mathcal{C}^{d f t}$.

Definition: The class $\mathcal{C}^{\mathrm{cm} f b}$ of $M$-channel orthonormal cosine-modulated FBs (CMFBs) is the one containing all FBs as in Fig. 1 where $H_{k}\left(e^{j \omega}\right)=P\left(e^{j(\omega-(k \pi / M)-(\pi / 2 M))}\right)+$ $P\left(e^{j(\omega+(k \pi / M)+(\pi / 2 M))}\right)$ for some filter $P\left(e^{j \omega}\right)$ called the prototype. For example, any $P\left(e^{j \omega}\right)$ having an alias-free $(2 M)$ support and with constant magnitude on its support is a valid prototype.

Theorem 5-PCFB Nonexistence for DFT, Cosine-Modulated FB Classes: There are families of input psds such that the class $\mathcal{C}^{d f t}$ defined above does not have a PCFB. The same holds for the class $\mathcal{C}^{\mathrm{cmfb}}$.

Proof: Consider first the class $\mathcal{C}^{d f t}$. Fig. 5(a) shows an input psd, two valid prototypes $P^{(j)}\left(e^{j \omega}\right)$, and the zeroth filters $H_{0}^{(j)}\left(e^{j \omega}\right)=P^{(j)}\left(e^{j \omega}\right), j=1,2$ in the DFT FBs produced by the prototypes. For the input psd, the filter $H_{0}^{(1)}\left(e^{j \omega}\right)$ produces the maximum subband variance achievable by any $M$-channel orthonormal $\mathrm{FB}$, and, hence, by any $\mathrm{FB}$ in $\mathcal{C}^{d f t}$. $\left(H_{0}^{(1)}\left(e^{j \omega}\right)\right.$ is the compaction filter [26] for the input psd.) Likewise, $H_{0}^{(2)}\left(e^{j \omega}\right)$ yields the minimum subband variance possible by any $M$-channel orthonormal FB, and, hence, by any FB in $\mathcal{C}^{d f t}$. Now, a PCFB simultaneously maximizes the largest and minimizes the least subband variance so that if a PCFB for $\mathcal{C}^{d f t}$ exists, it must contain both filters $H_{0}^{(j)}\left(e^{j \omega}\right), j=1,2$. This is impossible as these filters are not obtainable from each other by shift of an integer multiple of $2 \pi / M$; therefore, an FB having both of them cannot be in the class $\mathcal{C}^{d f t}$. Identical arguments hold for the class $\mathcal{C}^{c m f b}$, for the input psd, prototypes $P^{(j)}\left(e^{j \omega}\right)$, and corresponding filters $H_{0}^{(j)}\left(e^{j \omega}\right), j=1,2$, which are shown in Fig. 5(b). The only difference is that we no longer have $H_{0}^{(j)}\left(e^{j \omega}\right)=P^{(j)}\left(e^{j \omega}\right)$. In addition, it takes more effort to show that no FB in $\mathcal{C}^{\mathrm{cmfb}}$ can have both filters $H_{0}^{(j)}\left(e^{j \omega}\right), j=1,2$. We can show that if a CMFB has $H_{0}^{(1)}\left(e^{j \omega}\right)$ as one of its filters, then the band edges of all its filters must be multiples of $\pi / M$ so that $H_{0}^{(2)}\left(e^{j \omega}\right)$ cannot be a filter in it. In fact [4], a CMFB having $H_{0}^{(1)}\left(e^{j \omega}\right)$ of Fig. 5(b) as one of its filters is necessarily the CMFB produced by $P^{(1)}\left(e^{j \omega}\right)$ of Fig. 5(b) as a prototype.

\section{OPTIMAL NOISE REDUCTION WITH FILTERBANKS}

Suppose the FB input $x(n)$ of Fig. 1 is $x(n)=s(n)+\mu(n)$, where $s(n)$ is a pure signal, and $\mu(n)$ is zero mean additive noise. The desired FB output is $d(n)=s(n)$, and the goal of the system of Fig. 1 is to produce output $y(n)$ that approximates

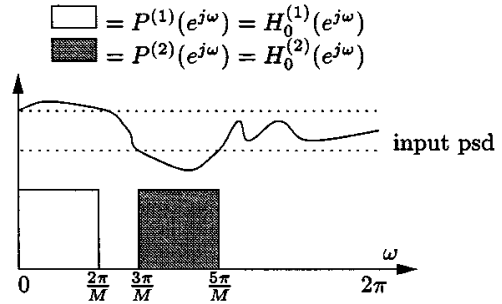

(a) Class of DFT FB's

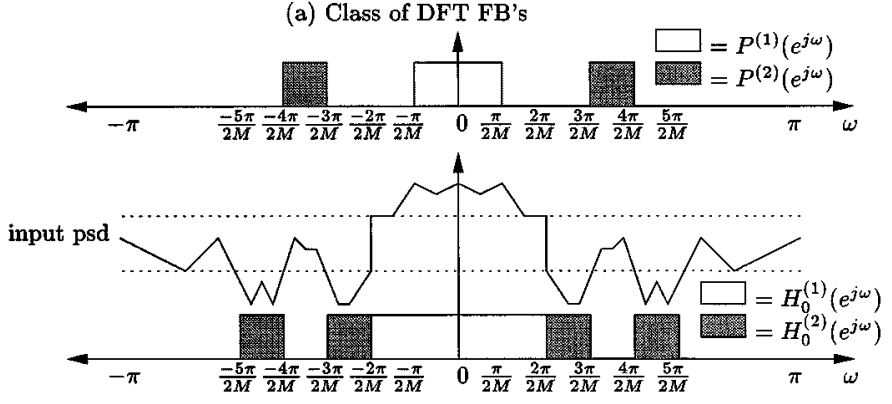

(b) Class of cosine-modulated FB's

Fig. 5. Nonexistence of PCFBs. (a) Class of DFT FBs. (b) Class of cosine-modulated FBs.

$s(n)$ as best as possible. We consider the case when all the subband processors $P_{i}$ are memoryless multipliers $k_{i}$, as shown in Fig. 6. This problem fits the formulation of Section II if we assume that $s(n)$ and $\mu(n)$ are uncorrelated and that $\mu(n)$ is white with a fixed known variance $\eta^{2}>0$. Indeed, using the notation of Section II, the $i$ th subband process $v_{i}^{(x)}(n)$ contains a signal component $v_{i}^{(s)}(n)$ and a zero mean additive noise component $v_{i}^{(\mu)}(n)$. Orthonormality of the FB ensures that the noise components are again white with variance $\eta^{2}$ and are uncorrelated to the signal components. The subband error process is

$$
\begin{aligned}
v_{i}^{(e)}(n) & =v_{i}^{(d)}(n)-v_{i}^{(y)}(n)=v_{i}^{(s)}(n)-k_{i} v_{i}^{(x)}(n) \\
& =\left(1-k_{i}\right) v_{i}^{(s)}(n)-k_{i} v_{i}^{(\mu)}(n) .
\end{aligned}
$$

Thus, the $M$ processes $v_{i}^{(e)}(n)$ are jointly WSS, and since $v_{i}^{(\mu)}(n)$ is zero mean and uncorrelated to $v_{i}^{(s)}(n)$

$$
E\left[\left|v_{i}^{(e)}(n)\right|^{2}\right]=\left|1-k_{i}\right|^{2} \sigma_{i}^{2}+\left|k_{i}\right|^{2} \eta^{2}
$$

where $\sigma_{i}^{2}=E\left[\left|v_{i}^{(s)}(n)\right|^{2}\right]$ is the $i$ th signal subband variance. The best choice of multiplier $k_{i}$ [minimizing the error (21)] is the zeroth-order Wiener filter $k_{i}=\sigma_{i}^{2} /\left(\sigma_{i}^{2}+\eta^{2}\right)$. This is implementable in practice as $\eta^{2}$ is known, and $\sigma_{i}^{2}=E\left[\left|v_{i}^{(x)}(n)\right|^{2}\right]-$ $\eta^{2}$ can be estimated from the subband signal $v_{i}^{(x)}(n)$. With this choice, (21) becomes $E\left[\left|v_{i}^{(e)}(n)\right|^{2}\right]=\left(\sigma_{i}^{2} \eta^{2} /\left(\sigma_{i}^{2}+\eta^{2}\right)\right)$, which is as in (3) with

$$
h_{i}(x)=\frac{x \eta^{2}}{x+\eta^{2}}
$$

This function $h_{i}$ is plotted in Fig. 7 and is easily verified to be concave on $[0, \infty)$. Therefore, by Theorem 3 , PCFBs are optimal if the subband multipliers $k_{i}$ are zeroth-order Wiener filters. 


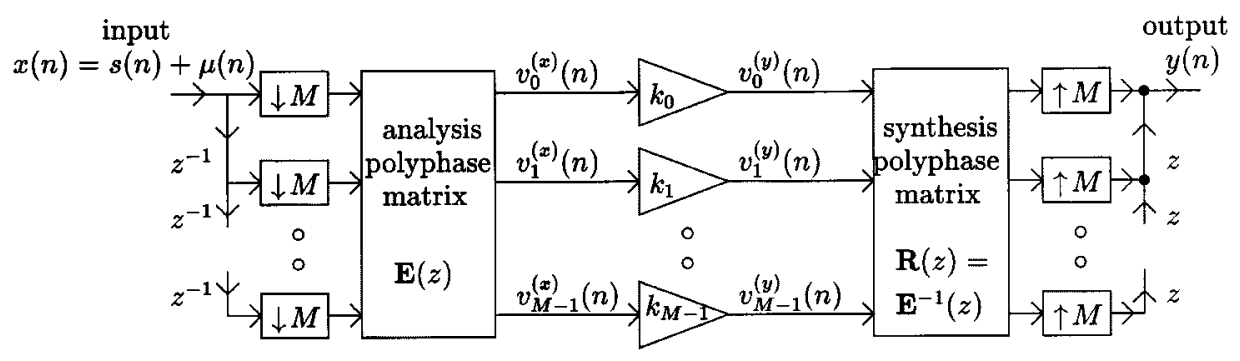

Fig. 6. FB-based noise reduction.

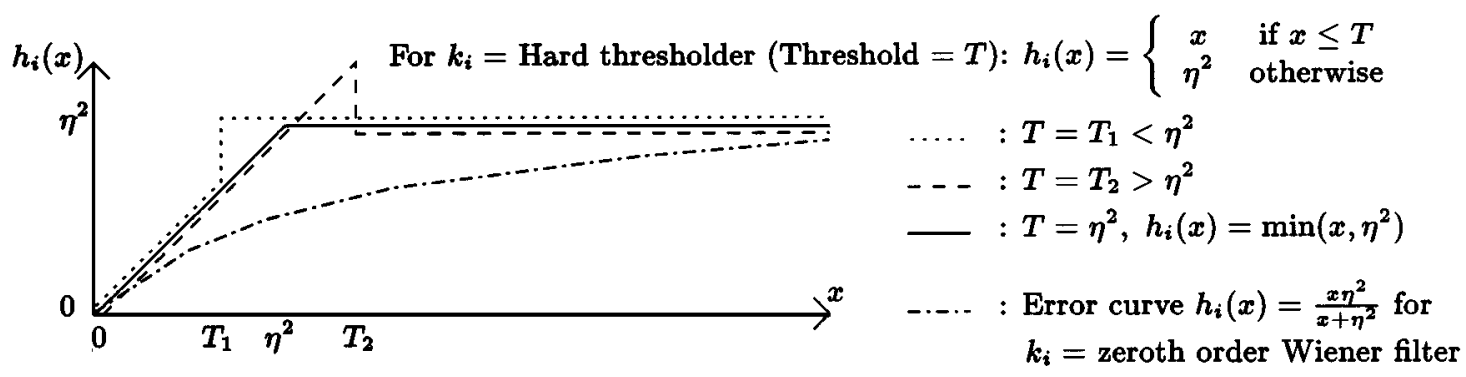

Fig. 7. Subband error functions in noise reduction.

\section{A. Remarks on PCFB Optimality for Noise Reduction}

PCFBs for the Pure or the Noisy Signal?: Notice a difference between the argument $\sigma_{i}^{2}$ of $h_{i}$ here and in (3). In (3), $\sigma_{i}^{2}$ was the variance of the subband signal $v_{i}^{(x)}(n)$ corresponding to the FB input $x(n)$. Here, it is the variance of the subband signal $v_{i}^{(s)}(n)=v_{i}^{(x)}(n)-v_{i}^{(\mu)}(n)$ corresponding to the pure signal $s(n)$. Thus, use of Theorem 3 proves the optimality of a PCFB for the signal $s(n)$, i.e., an FB that causes the subband variance vector corresponding to $s(n)$ to majorize the variance vectors obtained by using other FBs in the given class $\mathcal{C}$. However, because $v_{i}^{(\mu)}(n)$ is white with variance $\eta^{2}$ and uncorrelated to $v_{i}^{(s)}(n)$, we have $E\left[\left|v_{i}^{(s)}(n)\right|^{2}\right]=\sigma_{i}^{2}=E\left[\left|v_{i}^{(x)}(n)\right|^{2}\right]-\eta^{2}$. Thus, any PCFB for $s(n)$ is also a PCFB for $x(n)$ and vice versa.

Other Choices of Subband Multipliers: The Wiener filter is the optimum choice of the multiplier $k_{i}$ in Fig. 6. However, we may note that there are other choices that also result in an error function (21) that is concave in the subband variance $\sigma_{i}^{2}$. Thus, the PCFB will be optimal when the $k_{i}$ are any combination of such choices. One such other choice is a constant multiplier that is independent of the choice of FB (reminiscent of taps in a graphic equalizer in audio equipment). The error is then (21), which is, in fact, "linear" in $\sigma_{i}^{2}$. As the next remark shows, this observation yields an alternative proof of PCFB optimality with subband Wiener filtering. Another possible choice of multiplier $k_{i}$ is the subband hard threshold

$$
k_{i}=\left\{\begin{array}{ll}
1, & \text { if } \sigma_{i}^{2} \geq T \\
0, & \text { otherwise }
\end{array} .\right.
$$

The resulting subband error functions $h_{i}$ are plotted in Fig. 7 for different thresholds $T>0$. For the unique value $T=\eta^{2}$, which is the optimum threshold in the sense of minimizing $h_{i}(x)$ pointwise at all $x$, the resulting $h_{i}(x)=\min \left(x, \eta^{2}\right)$ is concave on $[0, \infty)$ (although not strictly concave) [23]. Unlike the Wiener filter, however, these choices of multiplier $k_{i}$ are of no serious practical interest and are mentioned here only to demonstrate an academic implication of PCFB optimality. More practical hard thresholding schemes for noise suppression [7] have a threshold that is applied individually to each element of the subband signal sequence (i.e., to each subband or "wavelet" coefficient) rather than on a subband by subband basis.

PCFB Optimality for Subband Wiener Filtering-Another Proof: One can prove PCFB optimality when all subband multipliers $k_{i}$ are Wiener filters without using any of the arguments of Section III involving majorization theory or the concavity of the function (22). To do this, observe that the PCFB is optimal if the subband multipliers are all constants independent of the FB. This was noted in the earlier remark and can be proved algebraically as in Section IV-C [see (19)] without using convexity theory. This is possible since the $h_{i}\left(\sigma_{i}^{2}\right)$ in this case are as in (21), which is "linear" (i.e., of the form $m_{i} \sigma_{i}^{2}+c_{i}$, where $m_{i}, c_{i}$ are constants). Since this optimality for constant multipliers holds irrespective of the multiplier values, it continues to hold if all these multipliers are optimized. Zeroth-order Wiener filters are the optimum multiplier choices, and hence, PCFBs are optimal when these are used in all subbands. This alternative proof fails, however, if some of the multipliers are not Wiener filters, e.g., they are other choices as mentioned in the earlier remark.

We summarize the above-mentioned results on PCFB optimality for noise reduction under Theorem 6 .

Theorem 6-Optimum FB-Based White Noise Suppression: In Fig. 6, let $s(n)$ be a $\operatorname{CWSS}(M)$ random process, and let $\mu(n)$ be zero mean additive white noise that has variance $\eta^{2}$ and is uncorrelated to $s(n)$. Let $\mathbf{v}=\left(\sigma_{0}^{2}, \sigma_{1}^{2}, \cdots, \sigma_{M-1}^{2}\right)^{T}$ denote the subband variance vector corresponding to $s(n)$. Let each subband multiplier $k_{i}$ be a zeroth-order Wiener filter $k_{i}=\sigma_{i}^{2} /\left(\sigma_{i}^{2}+\eta^{2}\right)$. Consider the FB optimization problem of minimizing the average mean square error between the FB output $y(n)$ and the desired signal $s(n)$. This is equivalent to minimizing $g(\mathbf{v})=(1 / M) \sum_{i=0}^{M-1} h_{i}\left(\sigma_{i}^{2}\right)$, where $h_{i}(x)=\left(x \eta^{2} /\left(x+\eta^{2}\right)\right)$. As these $h_{i}$ are all concave, a PCFB 


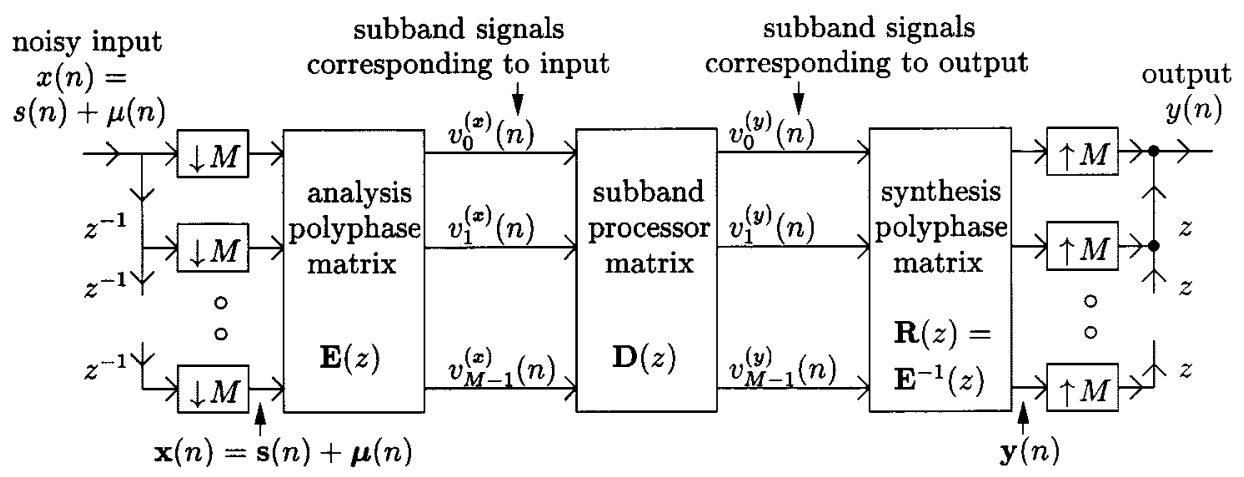

Fig. 8. Subband noise reduction: System of Section VI-B.

for $s(n)$ is optimal for this situation. This PCFB is also a PCFB for the input $x(n)$ since the noise is white. This optimality of the PCFB holds even with certain other choices of some or all of the subband multipliers $k_{i}$, namely subband hard thresholders (with threshold $\eta^{2}$ ) and constants (independent of choice of FB) since this merely changes the functional form of the corresponding $h_{i}$ but preserves its concavity.

\section{B. Subband Wiener Filtering: An Alternative Approach}

Since the subband processors studied above were LTI systems, it is possible to take a linear systems approach to the problem, as we elaborate here. While this approach does not prove Theorem 6 (derived above) in its entirety, it allows us to generalize some parts of its statement further. In particular, it allows certain extensions to cases when the noise is colored and the FBs are biorthogonal as opposed to orthonormal.

Consider the system of Fig. 8, where the boldface vectors $\mathbf{s}(n), \boldsymbol{\mu}(n), \mathbf{x}(n)$ and $\mathbf{y}(n)$ are all $M$-fold blocked versions of the corresponding scalar processes $s(n), \mu(n), x(n)$, and $y(n)$, and the $\mathrm{D}\left(e^{j \omega}\right)$ represents any $M \times M$ LTI system. We assume that $\mathbf{s}(n)$ and $\boldsymbol{\mu}(n)$ are uncorrelated WSS vector processes with psd matrices $\mathbf{S}_{\mathbf{s s}}\left(e^{j \omega}\right)$ and $\mathbf{S}_{\boldsymbol{\mu \mu}}\left(e^{j \omega}\right)$, respectively. The blocked version of the error is then $\mathbf{e}(n)=\mathbf{y}(n)-\mathbf{s}(n)$, which is WSS with psd matrix $\mathbf{S}_{\mathbf{e e}}\left(e^{j \omega}\right)$ as follows: (I denotes the identity matrix)

$$
\begin{aligned}
\mathbf{S}_{\mathbf{e e}}\left(e^{j \omega}\right)= & {\left[\mathbf{A}\left(e^{j \omega}\right)-\mathbf{I}\right] \mathbf{S}_{\mathbf{s s}}\left(e^{j \omega}\right)\left[\mathbf{A}\left(e^{j \omega}\right)-\mathbf{I}\right]^{\dagger} } \\
& +\mathbf{A}\left(e^{j \omega}\right) \mathbf{S}_{\boldsymbol{\mu \mu}}\left(e^{j \omega}\right) \mathbf{A}^{\dagger}\left(e^{j \omega}\right)
\end{aligned}
$$

where $\mathbf{A}\left(e^{j \omega}\right)=\mathbf{R}\left(e^{j \omega}\right) \mathbf{D}\left(e^{j \omega}\right) \mathbf{E}\left(e^{j \omega}\right)$, and of course

$$
\mathbf{R}\left(e^{j \omega}\right)=\mathbf{E}^{-1}\left(e^{j \omega}\right)
$$

To see this, note that $\mathbf{e}(n)=\mathbf{e}_{s}(n)+\mathbf{e}_{\mu}(n)$, where $\mathbf{e}_{s}(n), \mathbf{e}_{\mu}(n)$ are obtained by passing $\mathbf{s}(n), \boldsymbol{\mu}(n)$ through transfer matrices $\left[\mathbf{A}\left(e^{j \omega}\right)-\mathbf{I}\right]$ and $\mathbf{A}\left(e^{j \omega}\right)$, respectively. Since $\mathbf{s}(n), \boldsymbol{\mu}(n)$ are uncorrelated WSS, so are $\mathbf{e}_{s}(n), \mathbf{e}_{\mu}(n)$; thus, their sum is WSS with psd equal to the sum of their psds, and each is easy to compute. Note that (24) and (25) do not assume orthonormality of the FB [i.e., that $\mathbf{E}\left(e^{j \omega}\right)$ is unitary for all $\omega$ ] or whiteness of the noise [i.e., that $\mathbf{S}_{\boldsymbol{\mu} \boldsymbol{\mu}}\left(e^{j \omega}\right)$ is the identity matrix]. The average mean-square value of the error $e(n)$ is

$$
\begin{gathered}
\varepsilon=\frac{1}{M} \operatorname{trace}\left(\boldsymbol{\Sigma}_{\mathbf{e e}}\right), \quad \text { where } \boldsymbol{\Sigma}_{\mathbf{e e}}=\frac{1}{2 \pi} \int_{0}^{2 \pi} \mathbf{S}_{\mathbf{e e}}\left(e^{j \omega}\right) d \omega \\
=\text { autocorrelation matrix of } \mathbf{e}(n) .
\end{gathered}
$$

1) Memoryless $\mathbf{E}, \mathbf{D}, \mathbf{R}$ : If the transfer matrices $\mathbf{E}\left(e^{j \omega}\right), \mathbf{D}\left(e^{j \omega}\right)$ and $\mathbf{R}\left(e^{j \omega}\right)$ are all memoryless, then so is $\mathbf{A}=\mathbf{R D E}$, and

$$
\boldsymbol{\Sigma}_{\mathbf{e e}}=[\mathbf{A}-\mathbf{I}] \boldsymbol{\Sigma}_{\mathbf{s s}}[\mathbf{A}-\mathbf{I}]^{\dagger}+\mathbf{A} \boldsymbol{\Sigma}_{\boldsymbol{\mu \mu}} \mathbf{A}^{\dagger}
$$

where $\Sigma_{\mathrm{ss}}, \boldsymbol{\Sigma}_{\boldsymbol{\mu} \boldsymbol{\mu}}$ are autocorrelation matrices of $\mathrm{s}(n)$ and $\boldsymbol{\mu}(n)$, respectively. If $\mathbf{D}$ is unconstrained, so is $\mathbf{A}$, and the optimum $\mathbf{A}$ is simply the zeroth-order vector Wiener filter for the noisy input $\mathbf{x}(n)$, i.e.,

$$
\mathrm{A}=\mathrm{RDE}=\boldsymbol{\Sigma}_{\mathrm{ss}}\left[\boldsymbol{\Sigma}_{\mathrm{ss}}+\boldsymbol{\Sigma}_{\mu \mu}\right]^{-1}
$$

Suppose the signal and noise have a common KLT, i.e., for some unitary $\mathbf{T}$, both $\mathbf{T} \Sigma_{\mathbf{s s}} \mathbf{T}^{\dagger}=\boldsymbol{\Lambda}_{\mathbf{s s}}$ and $\mathbf{T} \boldsymbol{\Sigma}_{\boldsymbol{\mu \mu}} \mathbf{T}^{\dagger}=\boldsymbol{\Lambda}_{\boldsymbol{\mu} \boldsymbol{\mu}}$ are diagonal matrices. Then, substitution in (28) shows that $\mathbf{A}=$ $\mathbf{R D E}=\mathbf{T}^{\dagger} \mathbf{W} \mathbf{T}$, where $\mathbf{W}=\boldsymbol{\Lambda}_{\mathbf{s s}}\left[\boldsymbol{\Lambda}_{\mathbf{s s}}+\boldsymbol{\Lambda}_{\boldsymbol{\mu} \boldsymbol{\mu}}\right]^{-1}$ is diagonal. Therefore, the choice $\mathbf{E}=\mathbf{T}$ and $\mathbf{D}=\mathbf{W}$ is optimum under these conditions. Clearly, with this choice, the diagonal elements of the (diagonal) matrix $\mathbf{D}$ are the scalar zeroth-order Wiener filters for their corresponding inputs. Thus, we have proved the following theorem.

Theorem 7-Optimum Memoryless Transform for Subband Wiener Filtering: In Fig. 6, let the pure signal $s(n)$ and the zero mean additive noise $\mu(n)$ be uncorrelated $\operatorname{CWSS}(M)$ random processes. The noise $\mu(n)$ could be colored. Let all the subband multipliers $k_{i}$ be zeroth-order Wiener filters for reducing the noise component in their respective input. Suppose there is a common KLT for the signal and noise, namely, the unitary matrix $\mathbf{T}$. Then, the choice $\mathbf{E}(z)=\mathbf{T}$ in Fig. 6 gives optimum noise reduction among all choices where $\mathbf{E}(z)$ is a constant matrix. In other words, the common KLT is the optimum FB among all memoryless biorthogonal transforms in the sense of maximizing the output SNR.

Relation Between Theorems 6 and 7: Theorem 6 proves optimality of a PCFB for a general class $\mathcal{C}$ of orthonormal FBs for many white noise suppression problems where the subband multipliers could be any combination of Wiener filters, hard thresholds, and constants. On the other hand, Theorem 7 focuses on the case when all subband multipliers are Wiener filters, and on a special class of FBs, namely, the class $\mathcal{C}^{b}$ of all FBs with a constant (memoryless) polyphase matrix. Notice that $\mathcal{C}^{b}$ includes the orthogonal transform coder class $\mathcal{C}^{t}$. All Theorem 6 says about this case is that a signal KLT is the optimum FB within $\mathcal{C}^{t}$ when the noise is white. Notice that this FB is a 
common signal and noise KLT since any orthogonal transform is a KLT for a white input. Thus, Theorem 7 generalizes the result to the situation when the noise is colored and shows optimality of the common KLT among a larger class $\mathcal{C}^{b}$ of all memoryless biorthogonal transforms. In summary, Theorems 6 and 7 have a common element, which they generalize in different directions.

Further Generalizations: Attempts to combine Theorems 6 and 7 yield many interesting further generalizations and open problems. For example, let us restrict attention to orthogonal transforms in Theorem 7. The common signal and noise PCFB (KLT), if it exists, can then be shown to be optimal even if the subband multipliers are any combination of Wiener filters, hard thresholds, and constants (as opposed to all being Wiener filters as in Theorem 7). This result is shown in [3], using the convexity of certain search spaces associated with the signal and noise spectrum. As the input noise is colored, the subband noise variances are no longer constant but depend on choice of FB; hence, the approach used to prove Theorem 6 needs some modifications, as shown in [3]. It also appears plausible that the above optimality of the common KLT extends to the class of all memoryless biorthogonal transforms. ${ }^{4}$ Verifying this is, however, currently an open problem.

2) Case When E, D, R Have Memory-Higher Order Subband Wiener Filters: Suppose the LTI systems E, D, R in Fig. 8 have memory. The FB optimization problem then involves choosing from the given class of analysis polyphase matrices $\mathbf{E}\left(e^{j \omega}\right)$ the one minimizing the error $\varepsilon$ of (26) where $\mathbf{S}_{\mathbf{e e}}\left(e^{j \omega}\right)$ is as in (24) and (25), and $\mathbf{D}\left(e^{j \omega}\right)$ is an appropriately constrained matrix. For example, if $N$ th-order Wiener filters are used in all subbands, then $\mathrm{D}\left(e^{j \omega}\right)$ is a diagonal matrix depending in an involved manner on $\mathbf{E}\left(e^{j \omega}\right)$. The FB optimization for such cases appears to be extremely involved, and no analytical results are known to the authors at this time. $N$ th-order Wiener filters $(N>0)$ cannot be handled like zeroth-order ones as in Section VI-A. This is because the minimization objective now depends on not just the subband variances but on $N$ more coefficients in the autocorrelation sequences of the subband random processes.

If ideal Wiener filters are used in each subband, an analog of Theorem 7 can be stated. In this case, any orthonormal FB whose polyphase matrix $\mathbf{E}\left(e^{j \omega}\right)$ diagonalizes both the signal and noise psd matrices is optimal over the class of all unconstrained biorthogonal FBs. This result is obtained by repeating the methods used to prove Theorem 7 at each frequency $\omega$. We may note that the optimal FB mentioned here need not be a PCFB for either the signal or the noise. Diagonalization of the psd matrices is sufficient, and there is no constraint on the ordering of the subband spectra. However, this result is not very interesting when the scalar signal and noise input to the FB are WSS [as opposed to CWSS $(M)$ ]. In this case, diagonalization of psd matrices is trivial using any orthonormal FB with nonoverlapping analysis filters. The resulting system is then equivalent to an ideal scalar Wiener filter acting directly on the scalar input without use of any FB.

\footnotetext{
${ }^{4} \mathrm{An}$ analogous result is true for the high bitrate coding problem with optimal bit-allocation (Section IV-C), i.e., the signal KLT is optimal over all memoryless biorthogonal transforms. This is proved using the Hadamard inequality for determinants.
}

\section{CONCLUSION}

We have pointed out a strong connection between the optimization of orthonormal filterbanks and the principal component property. The main result is that a principal component filterbank (PCFB) is optimal whenever the minimization objective is a concave function of the vector consisting of the subband variances of the FB. We have shown various signal processing systems in which the FB optimization involves minimizing such a concave objective. In particular, the known results on optimality of PCFBs for compression can be explained in this manner. PCFBs are also shown to be optimal for subband domain white noise suppression using any combination of zeroth-order Wiener filters and hard thresholds in the subbands. Some extensions have been made to biorthogonal FBs and to the case when the noise is colored. We have also shown that the classes of ideal DFT and cosine-modulated FBs do not have PCFBs.

A companion paper [3] contains further results on colored noise suppression. It proves optimality of the common signal and noise KLT among the orthogonal transform coder class for noise suppression using any combination of Wiener filters, hard thresholds, and constants as subband multipliers. This further generalizes some parts of Theorem 7 of the present work. It is also shown in [3] that an analogous result on optimality of a common signal and noise PCFB for the class $\mathcal{C}^{u}$ of unconstrained FBs is false. We study the effect of absence of a PCFB on the FB optimization and show that in general, the problem becomes analytically intractable. We examine the connection between compaction filters, PCFBs, and FB optimization. Extensions of the PCFB concept to classes of nonuniform FBs have been studied in [5]. We have also shown [28] that PCFBs are optimal for maximizing the bit rate or minimizing the power requirement in discrete multitone (DMT) communication systems [13], again due to concavity of the relevant minimization objectives.

\section{APPENDIX A}

\section{DOUbly STOCHASTIC MATRICES}

Here, we prove that all convex combinations and products of $M \times M$ doubly stochastic matrices are also doubly stochastic. It suffices to prove this for two matrices since we can continue by induction. Define the vector $\mathbf{k} \in \mathcal{R}^{M}$ as $\mathbf{k}=(1,1, \cdots, 1)^{T}$. Then, by definition, an $M \times M$ matrix $\mathbf{Q}$ is doubly stochastic iff all its entries are non-negative, $\mathbf{Q k}=\mathbf{k}$, and $\mathbf{k}^{T} \mathbf{Q}=\mathbf{k}^{T}$. Now, consider a convex combination $\mathbf{C}=\alpha \mathbf{A}+(1-\alpha) \mathbf{B}$ (where $0 \leq \alpha \leq 1)$ and a product $\mathbf{D}=\mathbf{A B}$ of the $M \times M$ doubly stochastic matrices $\mathbf{A}$ and $\mathbf{B}$. It is required to show that $\mathbf{C}, \mathbf{D}$ are doubly stochastic. Clearly, since $\mathbf{A}, \mathbf{B}$ have non-negative entries, so do $\mathbf{C}, \mathbf{D}$. The proof is then completed by (29), shown at the top of the next page. It also shows that the set of all $M \times M$ doubly stochastic matrices is convex.

\section{APPENDIX B}

\section{ARe Doubly Stochastic Matrices ORThostochastic?}

Evidently, every $M \times M$ orthostochastic matrix is doubly stochastic. Here, we show that the converse is true if $M \leq 2$ 


$$
\begin{aligned}
& \mathbf{C k}=\alpha \mathbf{A k}+(1-\alpha) \mathbf{B k}=\alpha \mathbf{k}+(1-\alpha) \mathbf{k}=\mathbf{k}, \quad \text { and similarly } \quad \mathbf{k}^{T} \mathbf{C}=\mathbf{C} \\
& \text { Likewise, } \quad \mathbf{D k}=\mathbf{A B k}=\mathbf{A k}=\mathbf{k}, \quad \text { and similarly } \quad \mathbf{k}^{T} \mathbf{D}=\mathbf{D}
\end{aligned}
$$

but is false if $M>2$. The case $M=1$ is trivial. For $M=2$, a $2 \times 2$ doubly stochastic matrix must have form $\mathbf{Q}=\left[\begin{array}{cc}p & 1-p \\ 1-p & p\end{array}\right]$ with $0 \leq p \leq 1$. Now, $p=\cos ^{2}(\theta)$ for some real $\theta$ so that $\stackrel{p}{\mathbf{Q}}$ is indeed the orthostochastic matrix corresponding to the unitary matrix $\left[\begin{array}{rr}\cos (\theta) & \sin (\theta) \\ -\sin (\theta) & \cos (\theta)\end{array}\right]$. For $M=3$, take the doubly stochastic matrix

$$
\mathbf{A}=\frac{1}{2}\left[\begin{array}{lll}
1 & 1 & 0 \\
1 & 0 & 1 \\
0 & 1 & 1
\end{array}\right]
$$

If $\mathbf{A}$ was the orthostochastic matrix corresponding to $\mathbf{U}$, then

$$
\mathbf{U}=\left[\begin{array}{lll}
a & b & 0 \\
c & 0 & d \\
0 & e & f
\end{array}\right]
$$

for some nonzero $a, b, c, d, e, f$. Thus, $\mathbf{U}$ cannot be unitary as no two of its rows can be orthogonal to each other. Therefore, $\mathbf{A}$ is not orthostochastic. Small perturbations of the entries of $\mathbf{A}$ can create other such examples. The doubly stochastic matrix $\left[\begin{array}{cc}\mathbf{A} & \mathbf{0} \\ \mathbf{0} & \mathbf{I}\end{array}\right]$ gives examples for $M>3$, where $\mathbf{0}, \mathbf{I}$ are, respectively, the zero and identity matrices of suitable size. This concludes the proof. We may note here that the set $\mathcal{O}_{M}$ of $M \times M$ orthostochastic matrices is convex if $M \leq 2$ (as it is then the set of $M \times M$ doubly stochastic matrices) but is not convex if $M>2$. This is because all $(M \times M)$ permutation matrices are in $\mathcal{O}_{M}$, and every doubly stochastic matrix is a convex combination of these matrices (Birkhoff's theorem). Therefore, if $\mathcal{O}_{M}$ were convex, it would contain all doubly stochastic matrices, but it does not if $M>2$.

\section{ACKNOWLEDGMENT}

The authors gratefully thank the reviewers for their many useful suggestions that improved the paper significantly. They are also thankful to Prof. S. Dasgupta for bringing to their attention the notion of Schur concavity [18].

\section{REFERENCES}

[1] S. Akkarakaran and P. P. Vaidyanathan, "On optimization of filter banks with denoising applications," in Proc. IEEE ISCAS, Orlando, FL, June 1999.

[2] — , "Optimized orthonormal transforms for SNR improvement by subband processing," in Proc. IEEE Workshop Signal Process. Adv. Wireless Commun., Annapolis, MD, May 1999.

[3] — , "Results on principal component filter banks: Colored noise suppression and existence issues," IEEE Trans. Inform. Theory, to be published.

[4] — - "Principal component filter banks: Existence issues, and application to modulated filter banks," in Proc. IEEE ISCAS, Geneva, Switzerland, May 2000.

[5] — - "On nonuniform principal component filter banks: Definitions, existence and optimality," Proc. SPIE, 2000.

[6] J. B. Conway, A Course in Functional Analysis. New York: SpringerVerlag, 1985.
[7] D. L. Donoho and I. M. Johnstone, "Ideal spatial adaptation by wavelet shrinkage," Biometrika, vol. 81, no. 3, pp. 425-455, 1994.

[8] N. Dunford and J. T. Schwartz, Linear Operators. New York: Interscience, 1964, vol. I and II.

[9] H. Feng and M. Effros, "On the rate-distortion optimality and computational efficiency of the Karhunen Loeve transform for lossy data compression,", preprint, to be published.

[10] G. H. Hardy, J. E. Littlewood, and G. Polya, Inequalities. Cambridge, U.K.: Cambridge Univ. Press, 1934.

[11] R. A. Horn and C. R. Johnson, Matrix Analysis. Cambridge, U.K.: Cambridge Univ. Press, 1985.

[12] Y. Huang and P. M. Schultheiss, "Block quantization of correlated Gaussian random variables," IEEE Trans. Commun. Syst., vol. C-10, pp. 289-296, Sept. 1963.

[13] I. Kalet, "The multitone channel," IEEE Trans. Commun., vol. 37, pp. 119-124, Feb. 1989.

[14] A. Kirac and P. P. Vaidyanathan, "Optimality of orthonormal transforms for subband coding," in Proc. IEEE DSP Workshop, Bryce Canyon, Utah, Aug. 1998.

[15] - "On existence of FIR principal component filter banks," in Proc. ICASSP, Seattle, WA, May 1998.

[16] S. Lang, Real Analysis. Reading, MA: Addison-Wesley, 1983.

[17] S. Mallat, A Wavelet Tour of Signal Processing. New York: Academic, 1998.

[18] A. W. Marshall and I. Olkin, Inequalities: Theory of Majorization and its Applications. New York: Academic, 1979.

[19] P. Moulin and M. K. Mihcak, "Theory and design of signal-adapted FIR paraunitary filter banks," IEEE Trans. Signal Processing, vol. 46, pp. 920-929, Apr. 1998.

[20] P. Moulin, M. Anitescu, and K. Ramchandran, "Theory of rate-distortion optimal, constrained filter banks-Application to IIR and FIR biorthogonal designs," IEEE Trans. Signal Processing, pp. 1120-1132, Apr. 2000.

[21] R. T. Rockafellar, Convex Analysis. Princeton, NJ: Princeton Univ. Press, 1970.

[22] W. Rudin, Functional Analysis. New York: McGraw-Hill, 1991.

[23] D. L. Russell, Optimization Theory. New York: W. A. Benjamin, 1970.

[24] M. K. Tsatsanis and G. B. Giannakis, "Principal component filter banks for optimal multiresolution analysis," IEEE Trans. Signal Processing, vol. 43, pp. 1766-1777, Aug. 1995.

[25] M. Unser, "An extension of the KLT for wavelets and perfect reconstruction filter banks," in Proc. SPIE 2034, Wavelet Appl. Signal Image Process., San Diego, CA, 1993, pp. 45-56.

[26] P. P. Vaidyanathan, "Theory of optimal orthonormal subband coders," IEEE Trans. Signal Processing, vol. 46, pp. 1528-1543, June 1998.

[27] P. P. Vaidyanathan and A. Kirac, "Results on optimal biorthogonal filter banks," IEEE Trans. Circuits Syst. II, vol. 45, pp. 932-947, August 1998.

[28] P. P. Vaidyanathan, Y.-P. Lin, S. Akkarakaran, and S.-M. Phoong, "Optimality of principal component filter banks for discrete multitone communication systems," in Proc. IEEE ISCAS, Geneva, Switzerland, May 2000 .

[29] S. Akkarakaran, "Proof of majorization theorem,", [Online] Available http://www.systems.caltech.edu/dsp/students/sony/journ/majproof.ps.

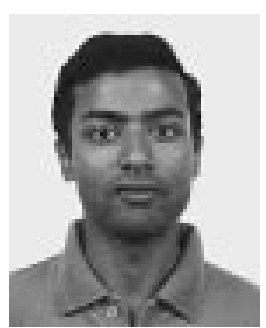

Sony Akkarakaran was born in Thrissur, India, in 1975. He received the B.Tech. degree from the Indian Institute of Technology, Bombay, in 1996, and the M.S. degree from the California Institute of Technology (Caltech), Pasadena, in 1997, both in electrical engineering. He is currently pursuing the $\mathrm{Ph} . \mathrm{D}$. degree in the field of digital signal processing at $\mathrm{Cal}$ tech. His research interests are multirate systems and wavelets and their communications applications. 


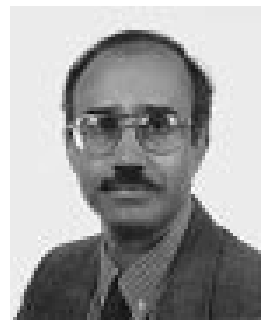

P. P. Vaidyanathan (S'80-M'83-SM'88-F'91) was born in Calcutta, India, on October 16, 1954. He received the B.Sc. (Hons.) degree in physics and the B.Tech. and M.Tech. degrees in radiophysics and electronics, all from the University of Calcutta, in 1974, 1977 and 1979, respectively, and the Ph.D. degree in electrical and computer engineering from the University of California, Santa Barbara (UCSB), in 1982.

He was a Post Doctoral Fellow at UCSB from September 1982 to March 1983. In March 1983, he joined the Electrical Engineering Department, California Institute of Technology (Caltech), Pasadena, as an Assistant Professor, and since 1993, he has been Professor of electrical engineering. His main research interests are in digital signal processing, multirate systems, wavelet transforms, and adaptive filtering. He has authored a number of papers in IEEE journals and is the author of the book Multirate Systems and Filter Banks (Englewood Cliffs, NJ: Prentice-Hall, 1993). He has written several chapters for various signal processing handbooks. He is a Consulting Editor for the Applied and Computational Harmonic Analysis.

Dr. Vaidyanathan served as Vice-Chairman of the Technical Program Committee for the 1983 IEEE International Symposium on Circuits and Systems and as the Technical Program Chairman for the 1992 IEEE International Symposium on Circuits and Systems. He was an Associate Editor for the IEEE TRANSACTIONS ON CIRCUITS AND SySTEMS from 1985 to 1987 and is currently an Associate Editor for the IEEE Signal Processing LeTters. He was a Guest Editor for special issues of the IEEE TRANSACTIONS ON Signal Processing and IEEE TRANSACtions ON Circuits AND Systems II on the topics of filter banks, wavelets, and subband coders in 1998. He was a recipient of the Award for excellence in teaching at the California Institute of Technology for 1983-1984, 1992-1993, and 1993-1994. He also received the NSF's Presidential Young Investigator Award in 1986. In 1989, he received the IEEE ASSP Senior Award for his paper on multirate perfect-reconstruction filter banks. In 1990, he was the recipient of the S. K. Mitra Memorial Award from the Institute of Electronics and Telecommuncations Engineers, India, for his joint paper in the IETE journal. He was also the coauthor of a paper on linear-phase perfect reconstruction filter banks in the IEEE TRANSACTIONS ON Signal PROCESSING TRANSACTIONS, for which the first author (T. Nguyen) received the Young Outstanding Author Award in 1993. He received the 1995 F. E. Terman Award of the American Society for Engineering Education, sponsored by Hewlett Packard Co., for his contributions to engineering education, especially the book Multirate Systems and Filter Banks. He has given several plenary talks at the Eusipco'98, Asimolar'88, and SPCOM'95 conferences on signal processing. He was a Distinguished Lecturer for the IEEE Signal Processing Society for the year 1996-1997. In 1999, he received the IEEE CAS Society's Golden Jubilee Medal. 